SFB

On covariation estimation for

823 multivariate continuous Itô semimartingales with noise in non-synchronous observation schemes.

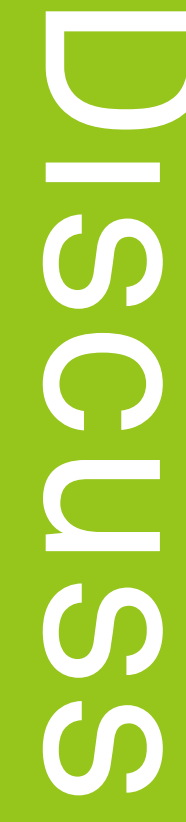

Kim Christensen, Mark Podolskij,

Mathias Vetter

Nr. 50/2011

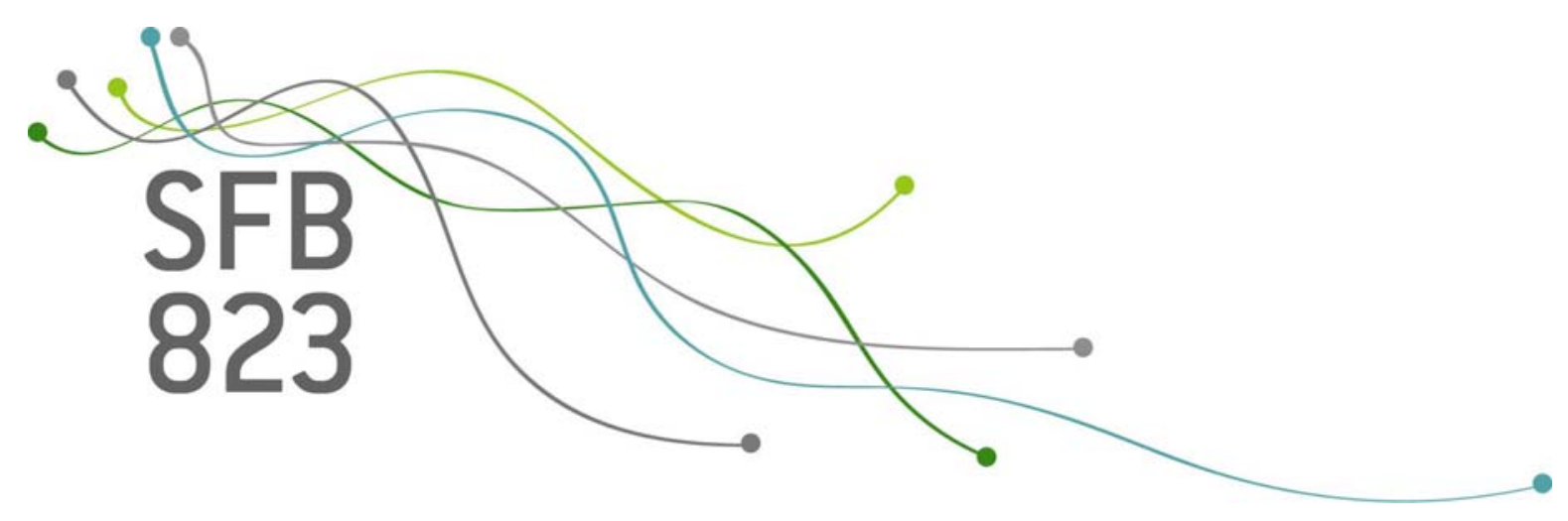





\title{
On covariation estimation for multivariate continuous Itô semimartingales with noise in non-synchronous observation schemes.
}

\author{
Kim Christensen * $^{*} \quad$ Mark Podolskij $^{\dagger} \quad$ Mathias Vetter ${ }^{\ddagger}$
}

December 3, 2011

\begin{abstract}
This paper presents a Hayashi-Yoshida type estimator for the covariation matrix of continuous Itô semimartingales observed with noise. The coordinates of the multivariate process are assumed to be observed at highly frequent nonsynchronous points. The estimator of the covariation matrix is designed via a certain combination of the local averages and the Hayashi-Yoshida estimator. Our method does not require any synchronization of the observation scheme (as e.g. previous tick method or refreshing time method) and it is robust to some dependence structure of the noise process. We show the associated central limit theorem for the proposed estimator and provide a feasible asymptotic result. Our proofs are based on a blocking technique and a stable convergence theorem for semimartingales. Finally, we show simulation results for the proposed estimator to illustrate its finite sample properties.
\end{abstract}

Keywords: central limit theorem, Hayashi-Yoshida estimator, high frequency observations, Itô semimartingale, pre-averaging, stable convergence.

AMS 2000 subject classifications. Primary 62M09, 60F05, 62H12; secondary 62G20, 60G44.

\section{Introduction}

In the past years there has been a considerable development of statistical methods for stochastic processes observed at high frequency. This was mainly motivated by financial applications, where the data, such as stock prices or currencies,

\footnotetext{
${ }^{*}$ CREATES, University of Aarhus, Building 1326, 8000 Aarhus, Denmark, Email: kchristensen@creates.au.dk.

${ }^{\dagger}$ Department of Mathematics, Heidelberg University, INF 294, 69120 Heidelberg, Germany, Email: m.podolskij@uni-heidelberg.de.

${ }^{\ddagger}$ Ruhr-Universität Bochum, Fakultät für Mathematik, 44780 Bochum, Germany, Email: mathias.vetter@rub.de. His work is supported by Sonderforschungsbereich "Statistical modelling of nonlinear dynamic processes" (SFB 823) of the Deutsche Forschungsgemeinschaft.
} 
are observed very frequently. It is well known that under the no-arbitrage assumption price processes must follow a semimartingale (see e.g. [9]). However, at ultra high frequencies the financial data is contaminated by microstructure noise such as rounding errors, bid-ask bounds and misprints. This fact prevents us from using classical power variation based methods (see e.g. [2] or [14] among many others) to infer the characteristics of a semimartingale.

A standard model for a continuous Itô semimartingale observed with errors is given by

$$
Y_{t}=X_{t}+\varepsilon_{t}, \quad t \geq 0
$$

where $\left(X_{t}\right)_{t \geq 0}$ is a $d$-dimensional process (true price) of the form

$$
X_{t}=X_{0}+\int_{0}^{t} a_{s} d s+\int_{0}^{t} \sigma_{s} d W_{s}, \quad t \geq 0
$$

with $\left(a_{s}\right)_{s \geq 0}$ being an $\mathbb{R}^{d}$-valued càglàd process, $\left(\sigma_{s}\right)_{s \geq 0}$ being an $\mathbb{R}^{d \times d^{\prime}}$-valued càglàd volatility and $W$ representing a $d^{\prime}$-dimensional Brownian motion, and the $d$-dimensional error process $\varepsilon$ (microstructure noise) is iid with

$$
\mathbb{E}\left[\varepsilon_{t}\right]=0, \quad \mathbb{E}\left[\varepsilon_{t} \varepsilon_{t}^{\star}\right]=\Psi \in \mathbb{R}^{d \times d}
$$

independent of $X$. Throughout this work an asterisk denotes the transpose of a matrix.

The aim of this paper is to estimate the covariation matrix of $X$ over some interval, say $[0,1]$, i.e.

$$
[X]=\int_{0}^{1} \Sigma_{s} d s \in \mathbb{R}^{d \times d}, \quad \Sigma_{s}=\sigma_{s} \sigma_{s}^{\star}
$$

based on non-synchronous noisy observations $\left(Y=\left(Y^{1}, \ldots, Y^{d}\right)\right)$

$$
Y_{t_{i}^{k}}^{k}, \quad k=1, \ldots, d, \quad i=0, \ldots, n_{k},
$$

where $0=t_{0}^{k}<\cdots<t_{n_{k}}^{k}=1$ are partitions of the interval $[0,1]$ with $\max _{1 \leq i \leq n_{k}}\left|t_{i}^{k}-t_{i-1}^{k}\right| \rightarrow 0$ as $n_{k} \rightarrow \infty$ for all $1 \leq k \leq d$. The univariate counterpart of this problem has been studied intensively in the literature. Let us mention the two-scale approach of [24] (see [23] for its more efficient multi-scale version), the realised kernel method proposed in [3] and the pre-averaging concept originally introduced in [21] (and further studied in [15], [16], [20] in various settings) among others. These methods can be extended to the multivariate case in a rather straightforward manner if the observations are synchronous.

When the underlying data is non-synchronous, things are less obvious, as we are faced with two challenges at the same time: We have to de-noise the data as before, but we also need to apply a certain synchronization technique to create a new set of observations from which appropriate estimators for $[X]$ can be computed. For the multivariate realised kernel 
method, [4] proposed to cope with non-synchronous data by applying the refreshing time method first, which synchronizes the observations via a previous tick method. In a second step, a noise robust estimator is constructed from this new data set. Similar in spirit is the extension of the multi-scale estimator due to [6], where synchronous observations are obtained using the pseudo-aggregation algorithm of [19] first. The resulting covariance estimator then becomes a multi-scale version of the Hayashi-Yoshida estimator from [12], which originally has been introduced to deal with non-synchronicity in semimartingale models without noise.

Both approaches have their drawbacks, however: (a) Using the previous tick approach (which generates pseudo data points) may lead to inconsistent estimators for certain observation schemes; this phenomenon has been noticed in [12] in the setting of a pure diffusion; (b) After any of the synchronization techniques there remain at $\operatorname{most}^{\min _{1 \leq k \leq d}}\left(n_{k}\right)$ data points, which amounts in throwing away a lot of data. In the no-noise case, this is usually no problem, as for the Hayashi-Yoshida estimator exactly those observations are dropped that bear no additional information on the covariance, but for noisy data they still can be used to wipe out the noise.

To avoid these afore-mentioned drawbacks, we propose to combine a synchronization technique and a concept for de-noising as well, but in reverse order: We apply the pre-averaging approach, which is designed to locally diminish the influence of the noise, first, and use the Hayashi-Yoshida method afterwards. Our estimator, denoted by $H Y^{n}$, has the following important properties:

(i) In general, we use all observations $Y_{t_{i}^{k}}^{k}$;

(ii) The estimator has the optimal convergence rate $n^{-1 / 4}$;

(iii) The estimation method is robust to certain dependence structures of the noise process. This property is important for practical applications as the economic theory typically does not provide any insight on modeling the noise.

The main idea of the construction of $H Y^{n}$ comes from [7], where we indicated its consistency, but did not provide the complete asymptotic theory. In this paper we prove a stable central limit theorem for $H Y^{n}-[X]$ under very mild assumptions on the observation scheme $t_{i}^{k}$. Furthermore, we explain how to estimate the (random) asymptotic covariance matrix that appears in the central limit theorem to obtain a feasible result (which may be used in practice to construct confidence regions). We would like to emphasize again that the construction of our estimator is not completely obvious (as there are several ways of combining the Hayashi-Yoshida method and the pre-averaging approach, which may result 
in different properties) and that the proof of the main result, which is based on a certain blocking technique, martingale inequalities and a stable central limit theorem for semimartingales, is more advanced than in the univariate setting.

This paper is organized as follows: in Section 2 we introduce the set up and explain the construction of $H Y^{n}$. The main results of the paper including the consistency of $H Y^{n}$ and the associated stable central limit theorems are presented in Section 3. Section 4 deals with estimation techniques for the conditional variance, while in Section 5 we show some numerical results to illustrate the finite sample properties of our estimator. Section 6 is devoted to proofs, and some tedious parts are relegated to an Appendix in Section 7.

\section{The set up}

We start by introducing an appropriate filtered probability space on which our noisy process $Y$ is defined. Let $\left(\Omega^{(0)}, \mathcal{F}^{(0)},\left(\mathcal{F}_{t}^{(0)}\right)_{t \in[0,1]}, \mathbb{P}^{(0)}\right)$ be an arbitrary space on which the true price process $X$ lives, such that all involved process $a, \sigma$ and $W$ are adapted. Now we consider a second filtered probability space $\left(\Omega^{(1)}, \mathcal{F}^{(1)},\left(\mathcal{F}_{t}^{(1)}\right)_{t \in[0,1]}, \mathbb{P}^{(1)}\right)$, where $\Omega^{(1)}$ is the set of functions from $[0,1]$ to $\mathbb{R}^{d}$ and $\mathcal{F}^{(1)}$ is the Borel- $\sigma$-field on $\Omega^{(1)}$. We define on it the noise process $\varepsilon=\left(\varepsilon_{t}\right)_{t \in[0,1]}$ as follows: let $Q$ be a probability law on $\mathbb{R}^{d}$ (the marginal law of $\varepsilon$ ) and set $\mathbb{P}^{(1)}$ as $\mathbb{P}^{(1)}=\otimes_{t \in[0,1]} P_{t}$ with $P_{t}=Q$ for all $t \in[0,1]$. Now, $\left(\varepsilon_{t}\right)_{t \in[0,1]}$ is defined as the canonical process on $\left(\Omega^{(1)}, \mathcal{F}^{(1)},\left(\mathcal{F}_{t}^{(1)}\right)_{t \in[0,1]}, \mathbb{P}^{(1)}\right)$ with $\left(\mathcal{F}_{t}^{(1)}\right)_{t \in[0,1]}$ being the canonical filtration. The process $Y$ in $(1.1)$ lives on the product space $\left(\Omega, \mathcal{F},\left(\mathcal{F}_{t}\right)_{t \in[0,1]}, \mathbb{P}\right)$ given by:

$$
\Omega=\Omega^{(0)} \times \Omega^{(1)}, \quad \mathcal{F}=\mathcal{F}^{(0)} \times \mathcal{F}^{(1)}, \quad \mathcal{F}_{t}=\mathcal{F}_{t}^{(0)} \otimes \mathcal{F}_{t}^{(1)}, \quad \mathbb{P}=\mathbb{P}^{(0)} \otimes \mathbb{P}^{(1)}
$$

We remark that the probability space on which the process $\varepsilon$ lives is rather minimal; this is required for the stable convergence results. The process $Y$ is defined in continuous time just for convenience, although the mapping $(\omega, t) \rightarrow Y_{t}(\omega)$ is not $\mathcal{F} \otimes \mathcal{B}([0,1])$-measurable.

Now we introduce the assumptions on the sampling scheme.

Assumption (T): The observation times $t_{i}^{k}, i=0, \ldots, n_{k}, k=1, \ldots, d$ satisfy the following conditions:

(T1) (Time transformation) $t_{i}^{k}$ 's are transformations of an equidistant grid, i.e. there exist strictly monotonic (deterministic) functions $f_{k}:[0,1] \rightarrow[0,1]$ in $C^{1}([0,1])$ with non-zero right and left derivative in 0 and 1 , respectively, and 
with $f_{k}(0)=0, f_{k}(1)=1$ such that

$$
t_{i}^{k}=f_{k}^{-1}\left(i / n_{k}\right), \quad i=0, \ldots, n_{k}, \quad k=1, \ldots, d .
$$

(T2) (Boundedness of $f_{k}^{\prime}$ ) There exists a natural number $M>0$ such that

$$
M^{-1}<\sup _{x \in[0,1]}\left|f_{k}^{\prime}(x)\right|<M, \quad k=1, \ldots, d .
$$

(T3) (Comparable number of observations) Set $n=\sum_{k=1}^{d} n_{k}$. It holds that

$$
\frac{n_{k}}{n} \rightarrow m_{k} \in(0,1], \quad k=1, \ldots, d .
$$

(T4) (Joint grid points) The grids $\left(t_{i}^{k}\right),\left(t_{j}^{l}\right)(1 \leq k, l \leq d)$ have $n_{k l}$ common points which are denoted by $\left(t_{p}^{k l}\right)_{1 \leq p \leq n_{k l}}$. They have the representation $t_{p}^{k l}=f_{k l}^{-1}\left(p / n_{k l}\right)$ and $n_{k l} / n \rightarrow m_{k l} \in[0,1]$, where the functions $f_{k l}$ satisfy the same assumptions as $f_{k}$ in (T1) and (T2).

Let us shortly comment the above assumptions. Condition (T1) makes the explicit computation of the asymptotic covariance matrix in the forthcoming central limit theorem possible. Condition (T3) implies that the observation numbers $n_{k}$ have the same order. Condition (T2) means that the points of the lth grid do not lie dense between any two successive points of the $k$ th grid, i.e. the number of points $t_{j}^{l}$ that lie in the interval $\left[t_{i-1}^{k}, t_{i}^{k}\right]$ is uniformly bounded by a constant for all $1 \leq k, l \leq d$ (cf. Lemma 6.1 for a closely related result). When these last two conditions (similar number of observations and uniform boundedness of the number of points $t_{j}^{l}$ that belong to $\left[t_{i-1}^{k}, t_{i}^{k}\right]$ ) are fulfilled we say that the sampling schemes are comparable. Finally, condition (T4) means that the number of common points can be negligible compared to $n$ (if $m_{k l}=0$ ) or it can be of order $n$ (if $m_{k l}>0$ ).

We want to emphasize that the full force of Assumption (T) is only required for the proof of the central limit theorem! For the consistency result and the rate of convergence it suffices to assume that the grids $\left(t_{i}^{k}\right), k=1, \ldots, d$, are comparable. In particular, the representation (2.1) and the condition (T4) are not required.

Now we explain the construction of our estimator $H Y^{n}$. First, we choose a window size $k_{n}$ as

$$
k_{n}=\theta \sqrt{n}+o\left(n^{1 / 4}\right)
$$

for some constant $\theta>0$. In the next step we choose a positive weight function $g:[0,1] \rightarrow \mathbb{R}$ with $g(0)=g(1)=0$, which is piecewise $C^{1}$ with piecewise Lipschitz derivative $g^{\prime}$ and $\int_{0}^{1} g^{2}(x) d x>0$. For any $d$-dimensional stochastic process $V=\left(V^{1}, \ldots, V^{d}\right)$ we define the quantity

$$
\bar{V}_{t_{i}^{k}}^{k}=\sum_{j=1}^{k_{n}-1} g\left(\frac{j}{k_{n}}\right) \Delta_{t_{i+j}^{k}} V^{k}, \quad \Delta_{t_{i+j}^{k}} V^{k}=V_{t_{i+j}^{k}}^{k}-V_{t_{i+j-1}^{k}}^{k},
$$


which we call pre-averaging in tick time. The name refers to the fact that we use the same amount of data to construct $\bar{V}_{t_{i}^{k}}^{k}$ for all $1 \leq k \leq d$; alternatively one could perform the pre-averaging in calendar time by using the same time interval for all coordinates $V^{k}$, but with different number of observations in each time window. The latter approach would result in different properties of the estimator.

As discussed in [15], [16] or [21] the local averages technique performed in (2.4) diminishes the influence of the noise process $\varepsilon$ to some extent (but not completely) and helps us to get information about $\Sigma$. In the last step, as proposed in [7], we define a Hayashi-Yoshida type estimator based on pre-averaged observations by

$$
H Y_{k l}^{n}=\frac{1}{\left(\psi k_{n}\right)^{2}} \sum_{i=0}^{n_{k}-k_{n}+1} \sum_{j=0}^{n_{l}-k_{n}+1} \bar{Y}_{t_{i}^{k}}^{k} \bar{Y}_{t_{j}^{l}}^{l} 1_{\left\{\left(t_{i}^{k}, t_{i+k_{n}}^{k}\right] \cap\left(t_{j}^{l}, t_{j+k_{n}}^{l}\right] \neq \emptyset\right\}}
$$

with $\psi=\int_{0}^{1} g(x) d x$, and set $H Y^{n}=\left(H Y_{k l}^{n}\right)_{1 \leq k, l \leq d}$. In [7] we have already indicated the consistency of $H Y^{n}$. The aim of this paper is to provide the complete asymptotic theory to be able to construct confidence regions for the quadratic covariation $[X]$.

\section{The asymptotic theory}

We start with the consistency of the estimator $H Y^{n}$ which has been shown in [7].

Theorem 3.1 Assume that Assumption (T) holds and that the marginal law $Q$ of $\varepsilon$ has finite fourth moments. Then we have

$$
H Y^{n} \stackrel{\mathbb{P}}{\longrightarrow}[X]=\int_{0}^{1} \Sigma_{s} d s
$$

As we remarked above the full force of Assumption $(\mathrm{T})$ is not required for the proof of Theorem 3.1; it is just the comparability of sampling times which matters (see [7] for more details). Two remarks are in order.

Remark 3.2 (Univariate case) Even though no synchronization is necessary in the one-dimensional case, our estimator $H Y^{n}$ is for $d=d^{\prime}=1$ not identical to the univariate pre-averaged estimator proposed in [15]! Recall that the latter is defined as

$$
C^{n}=\frac{1}{k_{n}} \sum_{i=1}^{n-k_{n}+1}\left|\bar{Y}_{t_{i}}\right|^{2} \stackrel{\mathbb{P}}{\longrightarrow}[X] \int_{0}^{1} g^{2}(x) d x+\theta^{-2} \Psi \int_{0}^{1}\left(g^{\prime}(x)\right)^{2} d x
$$


where we set $t_{i}=t_{i}^{1}$. This should be compared to the univariate version of $H Y^{n}$, which is

$$
H Y^{n}=\frac{1}{\left(\psi k_{n}\right)^{2}} \sum_{i=k_{n}}^{n-2 k_{n}+1} \bar{Y}_{t_{i}}\left(\sum_{j=-k_{n}+1}^{k_{n}-1} \bar{Y}_{t_{i+j}}\right)
$$

plus some border terms of small order. We see immediately that the first estimator $C^{n}$ is biased (even after rescaling), where the bias is coming from $\Psi=\mathbb{E}\left[\varepsilon_{t}^{2}\right]$, while our estimator $H Y^{n}$ is unbiased. The reason for this is the additional averaging performed by $H Y^{n}$ (which is taken care by the second sum in the above formula). Indeed, the factor in front of $\varepsilon_{t_{i}}^{2}$ for $\frac{k_{n}}{n} \leq i \leq 1-\frac{k_{n}}{n}$ is equal to

$$
\left(\sum_{j=0}^{k_{n}-1} g\left(\frac{j+1}{k_{n}}\right)-g\left(\frac{j}{k_{n}}\right)\right)^{2}=(g(1)-g(0))^{2}=0,
$$

which explains why $\Psi$ does not appear in the limit of $H Y^{n}$. The unbiasedness of $H Y^{n}$ is an important feature as the estimation of the covariance matrix $\Psi$ of the noise can be problematic in practice, because we strongly rely on the iid assumption on the noise process to successfully perform the estimation of $\Psi$. Let us remark that pre-averaging in calendar time would also lead to a bias.

Remark 3.3 (m-dependent noise) Let us study the case of an $m$-dependent noise process. More precisely, we consider the multivariate discrete model $Y_{t_{i}^{k}}^{k}=X_{t_{i}^{k}}^{k}+\varepsilon_{t_{i}^{k}}^{k}, k=1, \ldots, d, i=0, \ldots, n_{k}$, where all previous assumptions are satisfied except the noise process is now $m$-dependent in tick time, which means that for $t_{i}^{k} \leq t_{j}^{l}$ the random variables $\varepsilon_{t_{i}^{k}}^{k}$ and $\varepsilon_{t_{j}^{l}}^{l}$ are independent, if $\left\|t_{i}^{k}-t_{j}^{l}\right\|>m$ with

$$
\left\|t_{i}^{k}-t_{j}^{l}\right\|=\min \left(j-\max \left\{z \mid t_{z}^{l} \leq t_{i}^{k}\right\}, \min \left\{z \mid t_{z}^{k} \geq t_{j}^{l}\right\}-i\right),
$$

and similarly for $t_{j}^{l}<t_{i}^{k}$. These types of models are important from the practical point of view. Our previous iid assumption on the noise process implies that $\varepsilon_{t_{i}^{k}}^{k}$ and $\varepsilon_{t_{j}^{l}}^{l}$ are possibly correlated when $t_{i}^{k}=t_{j}^{l}$; on the other hand they are independent even when the grid points $t_{i}^{k}$ and $t_{j}^{l}$ lie arbitrarily close, say less than a second apart. Such an assumption might be not very plausible from the finance point of view.

In the case of $m$-dependent noise the estimator $H Y^{n}$ still remains consistent, i.e. $H Y^{n}$ is robust to $m$-dependence in tick time. As in the previous remark only the products $\varepsilon_{t_{i}^{k}}^{k} \varepsilon_{t_{j}^{l}}^{l}$ with $\left\|t_{i}^{k}-t_{j}^{l}\right\| \leq m$ play a role when computing the bias. But these terms have asymptotically the same weight as for instance $\left(\varepsilon_{t_{i}^{k}}^{k}\right)^{2}$, which is 0 (see Remark 3.2). Thus, $H Y^{n}$ is unbiased.

In order to describe the weak limit associated with $H Y^{n}-[X]$ we need to introduce various notations. 
Notation. Let us first extend the weight function $g$ to the whole real line by setting $g(x)=0$ for $x \notin[0,1]$. We set for $x \in[0,1]$

$$
h_{k l}(x)=\frac{m_{k} f_{k}^{\prime}(x)}{m_{l} f_{l}^{\prime}(x)}, \quad 1 \leq k, l \leq d,
$$

where $f_{k}$ resp. $m_{k}$ are given in (2.1) resp. (2.2). Now we define two sets of functions, namely

$$
\left.\begin{array}{l}
\psi(s, x)=\int_{0}^{1} \int_{(u-1+s) x}^{1+x(s+u)} g(u) g(v) d v d u, \\
\bar{\psi}(s, x)=\int_{0}^{1} \int_{(u-1+s) x}^{1+x(s+u)} g(u) g^{\prime}(v) d v d u, \\
\widetilde{\psi}(s, x)=\int_{0}^{1} \int_{(u-1+s) x}^{1+x(s+u)} g^{\prime}(u) g^{\prime}(v) d v d u,
\end{array}\right\}
$$

and

$$
\left.\begin{array}{l}
\gamma_{k l, k^{\prime} l^{\prime}}(u)=\frac{1}{m_{l} f_{l}^{\prime}(u)} \int_{-\left(1+h_{l k}(u)\right)}^{1+h_{l k}(u)} \psi\left(s, h_{k l}(u)\right) \psi\left(h_{l^{\prime} l}(u) s, h_{k^{\prime} l^{\prime}}(u)\right) d s, \\
\bar{\gamma}_{k l, k^{\prime} l^{\prime}}(u)=\frac{m_{k k^{\prime}} f_{k k^{\prime}}^{\prime}(u)}{m_{l} f_{l}^{\prime}(u)} \int_{-\left(1+h_{l k}(u)\right)}^{1+h_{l k}(u)} \bar{\psi}\left(s, h_{k l}(u)\right) \bar{\psi}\left(h_{l^{\prime} l}(u) s, h_{k^{\prime} l^{\prime}}(u)\right) d s, \\
\widetilde{\gamma}_{k l, k^{\prime} l^{\prime}}(u)=\frac{m_{k k^{\prime}} f_{k k^{\prime}}^{\prime}(u) m_{l l^{\prime}} f_{l l^{\prime}}^{\prime}(u)}{m_{l} f_{l}^{\prime}(u)} \int_{-\left(1+h_{l k}(u)\right)}^{1+h_{l k}(u)} \widetilde{\psi}\left(s, h_{k l}(u)\right) \widetilde{\psi}\left(h_{l^{\prime} l}(u) s, h_{k^{\prime} l^{\prime}}(u)\right) d s,
\end{array}\right\}
$$

for $s \in \mathbb{R}, 1 \leq k, k^{\prime}, l, l^{\prime} \leq d$ and $u \in[0,1]$. Notice that when for example the number of joint points between the $k$ th and $k^{\prime}$ th grid is negligible compared to $n$ (which can only hold for $k \neq k^{\prime}$ ) then $m_{k k^{\prime}}=0$. In this case we have $\bar{\gamma}_{k l, k^{\prime} l^{\prime}} \equiv \widetilde{\gamma}_{k l, k^{\prime} l^{\prime}} \equiv 0$.

Before we present the stable central limit theorem let us recall the notion of stable convergence. A sequence of random variables $Z^{n}$ on $(\Omega, \mathcal{F}, \mathbb{P})$ converges stably in law towards $Z$, written $Z_{n} \stackrel{d_{s t}}{\longrightarrow} Z$, with $Z$ being defined on an extension $\left(\Omega^{\prime}, \mathcal{F}^{\prime}, \mathbb{P}^{\prime}\right)$ of the original probability space $(\Omega, \mathcal{F}, \mathbb{P})$, iff for any bounded, continuous real-valued function $g$ and any bounded $\mathcal{F}$-measurable random variable $V$ it holds that $\mathbb{E}\left[g\left(Z_{n}\right) V\right] \rightarrow \mathbb{E}^{\prime}[g(Z) V]$ as $n \rightarrow \infty$. We refer to [1], [22] or [17] for more details on stable convergence. The next theorem is the main result of our paper, and its proof is postponed to Section 6 .

Theorem 3.4 Assume that Assumption (T) holds and that the marginal law $Q$ of $\varepsilon$ has finite eighth moments. Then the sequence $L^{n}=n^{1 / 4}\left(H Y^{n}-[X]\right)$ converges stably in law towards a random variable $L$, defined on an extension $\left(\Omega^{\prime}, \mathcal{F}^{\prime}, \mathbb{P}^{\prime}\right)$ of the original probability space $(\Omega, \mathcal{F}, \mathbb{P})$, and L has a centered mixed normal distribution, i.e. conditionally on $\mathcal{F}, L=\left(L_{k l}\right)_{1 \leq k, l \leq d}$ has a centered normal distribution with

$$
\mathbb{E}^{\prime}\left[L_{k l} L_{k^{\prime} l^{\prime}} \mid \mathcal{F}\right]=V_{k l, k^{\prime} l^{\prime}}, \quad 1 \leq k, k^{\prime}, l, l^{\prime} \leq d,
$$


where the random variable $V_{k l, k^{\prime} l^{\prime}}$ is defined via

$$
\begin{aligned}
& V_{k l, k^{\prime} l^{\prime}}=\frac{1}{\psi^{4}} \int_{0}^{1}\left\{\theta\left(\gamma_{k l, k^{\prime} l^{\prime}}(u) \Sigma_{u}^{k k^{\prime}} \Sigma_{u}^{l l^{\prime}}+\gamma_{k l, l^{\prime} k^{\prime}}(u) \Sigma_{u}^{k l^{\prime}} \Sigma_{u}^{l k^{\prime}}\right)\right. \\
& +\theta^{-1}\left(\Psi^{l l^{\prime}} \bar{\gamma}_{l k, l^{\prime} k^{\prime}}(u) \Sigma_{u}^{k k^{\prime}}+\Psi^{l k^{\prime}} \bar{\gamma}_{l k, k^{\prime} l^{\prime}}(u) \Sigma_{u}^{k l^{\prime}}+\Psi^{k l^{\prime}} \bar{\gamma}_{k l, l^{\prime} k^{\prime}}(u) \Sigma_{u}^{l k^{\prime}}+\Psi^{k k^{\prime}} \bar{\gamma}_{k l, k^{\prime} l^{\prime}}(u) \Sigma_{u}^{l l^{\prime}}\right) \\
& \left.+\theta^{-3}\left(\Psi^{k k^{\prime}} \Psi^{l l^{\prime}} \widetilde{\gamma}_{k l, k^{\prime} l^{\prime}}(u)+\Psi^{k l^{\prime}} \Psi^{l k^{\prime}} \widetilde{\gamma}_{k l, l^{\prime} k^{\prime}}(u)\right)\right\} d u
\end{aligned}
$$

and the functions $\gamma_{k l, k^{\prime} l^{\prime}}, \bar{\gamma}_{k l, k^{\prime} l^{\prime}}, \widetilde{\gamma}_{k l, k^{\prime} l^{\prime}}$ are given by (3.3) and $\theta$ is defined in (2.3). We also write $L \sim M N(0, V)$ to denote the centered mixed normal distribution with random $\mathcal{F}$-measurable covariance matrix $V=\left(V_{k l, k^{\prime} l^{\prime}}\right)_{1 \leq k, k^{\prime}, l, l^{\prime} \leq d}$ above.

The rate of convergence $n^{-1 / 4}$ is known to be optimal for the parametric analogue of our estimation problem (i.e. when the process $\Sigma$ is constant); see e.g. [6] or [11]. We remark that the covariance matrix $\Psi$ of the noise process $\varepsilon$ always appears in the representation of $V$ as $\bar{\gamma}_{k k, k k}(u), \widetilde{\gamma}_{k k, k k}(u)>0$ for all $1 \leq k \leq d$.

Remark 3.5 (Univariate case) In the one-dimensional case $\left(d=d^{\prime}=1\right)$ we deduce that

$$
n^{1 / 4}\left(H Y^{n}-\int_{0}^{1} \sigma_{s}^{2} d s\right) \stackrel{d_{s t}}{\longrightarrow} M N(0, V),
$$

where the expression for $V$ simplifies to

$$
V=\frac{2}{\psi^{4}}\left(\theta \kappa \int_{0}^{1} \frac{\sigma_{u}^{4}}{f^{\prime}(u)} d u+2 \theta^{-1} \Psi \bar{\kappa} \int_{0}^{1} \sigma_{u}^{2} d u+\theta^{-3} \Psi^{2} \widetilde{\kappa}\right)
$$

with

$$
\kappa=\int_{-2}^{2} \psi^{2}(s, 1) d s, \quad \bar{\kappa}=\int_{-2}^{2} \bar{\psi}^{2}(s, 1) d s, \quad \widetilde{\kappa}=\int_{-2}^{2} \widetilde{\psi}^{2}(s, 1) d s
$$

Note that we have $f_{11}=f_{1}=: f, h_{11}=1$ and $m_{11}=m_{1}=1$, as well as $\int_{0}^{1} f^{\prime}(u) d u=1$. If we further deal with equidistant data it follows that $f(u)=u$.

To measure the quality of $H Y^{n}$ compared to alternative estimators in the one-dimensional setting, it is common to compute $V$ in the parametric model of zero drift and a constant volatility $\sigma$. In case of equidistant observations we know from [11] that the lower bound for the variance is then given by $8 \sigma^{3} \sqrt{\Psi}$. If we choose the (probably) simplest weight function given by $g(x)=\min (x, 1-x)$, some lengthy calculations give

$$
\kappa=\frac{7585}{1161216}, \quad \bar{\kappa}=\frac{151}{20160}, \quad \widetilde{\kappa}=\frac{1}{24}, \quad \psi=\frac{1}{4},
$$


and the optimal choice of $\theta$ corresponds to $\theta^{\star} \approx 2.381 \sqrt{\Psi} / \sigma$. Overall we obtain a minimal variance of $12.765 \sigma^{3} \sqrt{\Psi}$. This is quite close to the efficiency bound and also to the minimal variance of (the bias corrected version of) $C^{n}$, the original pre-averaged statistic for equidistant data from [15], which is about $8.545 \sigma^{3} \sqrt{\Psi}$. This mild loss in efficiency is the price we have to pay for the additional robustness property discussed in Remark 3.3.

\section{Estimation of variance}

To transform the probabilistic result of Theorem 3.4 into a feasible statistical one, we need to find a consistent estimator of the conditional covariance matrix $V$ defined by (3.4). We will introduce three different approaches to solve this task a general one, which works in arbitrary dimensions and does not require information of the time transforming functions; a second estimator, which uses local estimates of the volatility $\Sigma$; a third one tuned for the one-dimensional case, where the variance becomes particularly simple as seen in Remark 3.5. All proofs are given in Section 6.

Let us begin with the first estimator, for which we benefit from related work in [18], where an estimator for the variance of the usual Hayashi-Yoshida estimator in the no-noise case was constructed. We introduce a second auxiliary sequence $\beta_{n}=\varpi n^{\eta}+o\left(n^{\eta}\right), \varpi>0, \eta \in(0,1)$, and compute for each $\alpha \in\left\{0, \ldots\left[n / \beta_{n}\right]-1\right\}$ the statistic

$$
H Y_{k l}^{n}(\alpha)=\frac{1}{\left(\psi k_{n}\right)^{2}} \sum_{t_{i}^{k} \in B_{n}(\alpha)} \sum_{j=0}^{n_{l}-k_{n}+1} \bar{Y}_{t_{i}^{k}}^{k} \bar{Y}_{t_{j}^{l}}^{l} 1_{\left\{\left(t_{i}^{k}, t_{i+k_{n}}^{k}\right] \cap\left(t_{j}^{l}, t_{j+k_{n}}^{l}\right] \neq \emptyset\right\}},
$$

which is essentially the same quantity as $H Y_{k l}^{n}$, but we only sum over time points $t_{i}^{k}$ from the smaller interval $B_{n}(\alpha)=$ $\left[\frac{\alpha \beta_{n}}{n}, \frac{(\alpha+1) \beta_{n}}{n}\right)$. We set

$$
V_{k l, k^{\prime} l^{\prime}}^{n, 1}=\frac{\sqrt{n}}{2} \sum_{\alpha=1}^{\left[\frac{n}{\beta_{n}}\right]-1}\left(2 H Y_{k l}^{n}(\alpha) H Y_{k^{\prime} l^{\prime}}^{n}(\alpha)-H Y_{k l}^{n}(\alpha) H Y_{k^{\prime} l^{\prime}}^{n}(\alpha-1)-H Y_{k l}^{n}(\alpha-1) H Y_{k^{\prime} l^{\prime}}^{n}(\alpha)\right) .
$$

This estimator is based on a local estimation of the covariance of $H Y_{k l}^{n}$ and $H Y_{k^{\prime} l^{\prime}}^{n}$. In order to obtain reasonable estimates for this covariance on the interval $B_{n}(\alpha)$, we use $H Y_{k l}^{n}(\alpha) H Y_{k^{\prime} l^{\prime}}^{n}(\alpha)$ to mimic the covariance of interest plus the product of the expectations of both factors. The latter bias is corrected by quantities like $H Y_{k l}^{n}(\alpha) H Y_{k^{\prime} l^{\prime}}^{n}(\alpha-1)$, where we use the usual "conditional independence" of increments of $Y$ over disjoint intervals. $V_{k l, k^{\prime} l^{\prime}}^{n, 1}$ is now constructed as a symmetrized version of these local estimates, and we sum up over all $a$ afterwards to obtain a global one.

A drawback of this construction is that we need an additional condition on the process $\sigma$. In order for $H Y_{k l}^{n}(\alpha)$ and $H Y_{k l}^{n}(\alpha-1)$ to estimate the same quantity up to an error small enough, one usually postulates that $\sigma$ is an Itô semimartingale itself. Under a furher assumption on $\eta$ we have the following theorem. 
Theorem 4.1 Assume that Assumption (T) holds and that the marginal law $Q$ of $\varepsilon$ has finite eighth moments. Furthermore, suppose that $\sigma$ is a $d \times d^{\prime}$-semimartingale of the form (1.2) as well and let $1 / 2<\eta<2 / 3$. Then we have $V_{k l, k^{\prime} l^{\prime}}^{n, 1} \stackrel{\mathbb{P}}{\longrightarrow}$ $V_{k l, k^{\prime} l^{\prime}}$

As mentioned above, the second estimator uses local estimates of the volatility $\Sigma$ and the covariance matrix $\Psi$ of the noise, and we assume knowledge of the time-transforming functions $f_{k}$ and $f_{k l}$, which in practice have to be approximated via the observed time points.

We start with the construction of the estimator of $\Sigma_{s}$. We define $H Y^{n}([0, t])=\left(H Y_{k l}^{n}([0, t])\right)_{1 \leq k, l \leq d}$ for $t \in[0,1]$ by

$$
H Y_{k l}^{n}([0, t])=\frac{1}{\left(\psi k_{n}\right)^{2}} \sum_{i: t_{i+k_{n}}^{k} \leq t} \sum_{j: t_{j+k_{n}}^{l} \leq t} \bar{Y}_{t_{i}^{k}}^{k} \bar{Y}_{t_{j}^{l}}^{l} 1_{\left\{\left(t_{i}^{k}, t_{i+k_{n}}^{k}\right] \cap\left(t_{j}^{l}, t_{j+k_{n}}^{l}\right] \neq \emptyset\right\}}
$$

which is consistent for the integrated covariation matrix up to time $t$. As the volatility process $\left(\Sigma_{s}\right)_{s \in[0,1]}$ is leftcontinuous, it is a natural idea to estimate $\Sigma_{s}$ via

$$
\Sigma_{s, n}=\frac{H Y^{n}([0, s])-H Y^{n}\left(\left[0, s-l_{n}\right]\right)}{l_{n}}
$$

for some sequence $l_{n}$ with $l_{n} \rightarrow 0, \sqrt{n} l_{n} \rightarrow \infty$ and $s \in\left[l_{n}, 1\right]$ (for $s \in\left[0, l_{n}\right]$ we set $\Sigma_{s, n}=\Sigma_{l_{n}, n}$ ). The condition $\sqrt{n} l_{n} \rightarrow \infty$ is required to guarantee a sufficient amount of asymptotically uncorrelated summands in the definition of $\Sigma_{s, n}$.

The estimation of the covariance matrix $\Psi$ is somewhat easier. Recall that $\left(t_{p}^{k l}\right)_{1 \leq p \leq n_{k l}}$ denotes the set of common points of the $k$ th and the $l$ th grid, and define $i(p, k, l)=i$ with $t_{i}^{k}=t_{p}^{k l}$ for arbitrary $k, l=1, \ldots, d$. The estimator of $\Psi^{k l}$ is now given as

$$
\Psi_{n}^{k l}=-\frac{1}{n_{k l}} \sum_{p=1}^{n_{k l}} \Delta_{t_{i(p, k, l)}^{k}} Y^{k} \Delta_{t_{i(p, l, k)+1}^{l}} Y^{l} .
$$

The intuition behind this estimator is rather simple. First of all, since the increments of $X$ at highest frequency converge to 0 almost surely, the process $Y$ can be replaced by $\varepsilon$ without any changes in the limit. For this reason the estimator $\Psi_{n}^{k l}$ converges to $\Psi^{k l}$ almost surely by the strong law of large numbers (applied to the iid process $\varepsilon$ ) if $n_{k l} \rightarrow \infty$. When the sequence $n_{k l}$ does not diverge to $\infty$ then the convergence does not hold, but we have $n_{k l} / n \rightarrow m_{k l}=0$. Thus the corresponding functions $\bar{\gamma}$ and $\widetilde{\gamma}$ vanish as well, and this will be sufficient for the estimation of $V$.

After all we obtain the following result. 
Theorem 4.2 Assume that Assumption (T) holds and that the marginal law $Q$ of $\varepsilon$ has finite eighth moments. Then we have

$$
\begin{aligned}
& V_{k l, k^{\prime} l^{\prime}}^{n, 2}:=\frac{1}{\psi^{4}} \int_{0}^{1}\left\{\theta\left(\gamma_{k l, k^{\prime} l^{\prime}}(u) \Sigma_{u, n}^{k k^{\prime}} \Sigma_{u, n}^{l l^{\prime}}+\gamma_{k l, l^{\prime} k^{\prime}}(u) \Sigma_{u, n}^{k l^{\prime}} \Sigma_{u, n}^{l k^{\prime}}\right)\right. \\
& +\theta^{-1}\left(\Psi_{n}^{l l^{\prime}} \bar{\gamma}_{l k, l^{\prime} k^{\prime}}(u) \Sigma_{u, n}^{k k^{\prime}}+\Psi_{n}^{l k k^{\prime}} \bar{\gamma}_{l k, k^{\prime} l^{\prime}}(u) \Sigma_{u, n}^{k l^{\prime}}+\Psi_{n}^{k l^{\prime}} \bar{\gamma}_{k l, l^{\prime} k^{\prime}}(u) \Sigma_{u, n}^{l k^{\prime}}+\Psi_{n}^{k k^{\prime}} \bar{\gamma}_{k l, k^{\prime} l^{\prime}}(u) \Sigma_{u, n}^{l l^{\prime}}\right) \\
& \left.+\theta^{-3}\left(\Psi_{n}^{k k^{\prime}} \Psi_{n}^{l l^{\prime}} \widetilde{\gamma}_{k l, k^{\prime} l^{\prime}}(u)+\Psi_{n}^{k l^{\prime}} \Psi_{n}^{l k^{\prime}} \widetilde{\gamma}_{k l, l^{\prime} k^{\prime}}(u)\right)\right\} d u \stackrel{\mathbb{P}}{\longrightarrow} V_{k l, k^{\prime} l^{\prime}} .
\end{aligned}
$$

Let us finally focus on the one-dimensional case and recall the asymptotic variance in (3.5). As noted before, we do not have to care about any of the $\kappa$ 's from (3.6), as they can directly be computed from our choice of $g$. Using the univariate version of the estimator in (4.3) for $\Psi$ (which is consistent now) and the Hayashi-Yoshida type estimator $H Y^{n}$ for $\int_{0}^{1} \sigma_{u}^{2} d u$, all we need to find is a feasible estimator for the rescaled integrated quarticity $\int_{0}^{1} \frac{\sigma_{u}^{4}}{f^{\prime}(u)} d u$. Among several possibilities (including yet another Hayashi-Yoshida type one) we have decided to go with a pre-averaged version of realized quarticity. Thus we set

$$
\mu=\int_{0}^{1} g^{2}(u) d u, \quad \widetilde{\mu}=\int_{0}^{1}\left(g^{\prime}\right)^{2}(u) d u
$$

and define

$$
V^{n, 3}=\frac{2}{\psi^{4}}\left(\frac{\kappa}{3 \theta \mu^{2}} \sum_{i=1}^{n-k_{n}+1}\left|\bar{Y}_{t_{i}}\right|^{4}+\frac{2}{\theta} \Psi_{n} H Y^{n}\left(\bar{\kappa}-\frac{\kappa \widetilde{\mu}}{\mu}\right)+\frac{1}{\theta^{3}} \Psi_{n}^{2}\left(\widetilde{\kappa}-\frac{\kappa \widetilde{\mu}^{2}}{\mu^{2}}\right)\right) .
$$

The result precisely reads as follows.

Theorem 4.3 Let $d=1$ and assume that Assumption $(T)$ holds and that the marginal law $Q$ of $\varepsilon$ has finite eighth moments. Then we have $V^{n, 3} \stackrel{\mathbb{P}}{\longrightarrow} V$.

In order to present a feasible central limit theorem associated with Theorem 3.4 we vectorize the quantities $H Y^{n}$ and $[X]$, i.e.

$$
\widehat{H Y}^{n}=\operatorname{vec}\left(H Y^{n}\right), \quad \widehat{[X]}^{n}=\operatorname{vec}([X]),
$$

where vec is the vectorization operator that stacks columns of a matrix below one another, and set

$$
\begin{aligned}
\widehat{V}_{k l} & =V_{k-d[(k-1) / d],[(k-1) / d]+1, l-d[(l-1) / d],[(l-1) / d]+1}, \\
\widehat{V}_{k l}^{n, b} & =V_{k-d[(k-1) / d],[(k-1) / d]+1, l-d[(l-1) / d],[(l-1) / d]+1}^{n, b}
\end{aligned}
$$

with $1 \leq k, l \leq d^{2}$ and $b=1,2,3$. Now, the properties of stable convergence imply the following result, which can be directly applied for the construction of confidence regions. 
Corollary 4.4 Under the assumptions of Theorem 3.4 we obtain the stable convergence

$$
n^{1 / 4}\left(\widehat{H Y}^{n}-\widehat{[X]}\right) \stackrel{d_{s t}}{\longrightarrow} M N(0, \widehat{V}) .
$$

Also, for any $b=1,2,3$ and as long as the conditions for the corresponding theorem above are satisfied, we have the standard central limit theorem

$$
n^{1 / 4}\left(\widehat{V}^{n, b}\right)^{-1 / 2}\left(\widehat{H Y}^{n}-\widehat{[X]}\right) \stackrel{d}{\longrightarrow} N_{d^{2}}\left(0, I_{d^{2}}\right),
$$

where $N_{d^{2}}\left(0, I_{d^{2}}\right)$ denotes the $d^{2}$-dimensional normal distribution with covariance matrix equal to identity, and $\widehat{V}=$ $\left(\widehat{V}_{k l}\right)_{1 \leq k, l \leq d^{2}}, \widehat{V}^{n, b}=\left(\widehat{V}_{k l}^{n, b}\right)_{1 \leq k, l \leq d^{2}}$.

Remark 4.5 (m-dependent noise) We have indicated in Remark 3.3 that the consistency result for the Hayashi-Yoshida type estimator $H Y^{n}$ from Theorem 3.1 remains valid, if the assumption of independent noise variables is weakened to $m$-dependence. This does obviously not hold for the central limit theorem, as the particular form of the noise part of the asymptotic variance relies heavily on the independence assumption. Nevertheless, even in this framework a central limit theorem can be shown, but for the sake of brevity we dispense with the specification of its precise form. It is worth noticing, however, that $V_{k l, k^{\prime} l^{\prime}}^{n, 1}$ by construction remains a consistent estimator for the asymptotic variance in this rather general setting, as it is designed to mimic the covariance of of $H Y_{k l}^{n}$ and $H Y_{k^{\prime} l^{\prime}}^{n}$ without using any prior knowledge on $\varepsilon$ apart from dependence on only a finite number of neighbours. Therefore Theorem 4.1 and thus in turn (4.6) for $b=1$ hold true for $m$-dependent noise as well.

\section{Numerical study}

Here, we supplement the above asymptotic results based on $n \rightarrow \infty$ with a finite sample analysis by using Monte Carlo experiments. We simulate a bivariate stochastic volatility model with noise, as was also conducted in previous work of [4] and [7].

More specifically, to simulate efficient log-prices we consider

$$
\mathrm{d} X_{t}^{(i)}=a^{(i)} \mathrm{d} t+\rho^{(i)} \sigma_{t}^{(i)} \mathrm{d} B_{t}^{(i)}+\sqrt{1-\left[\rho^{(i)}\right]^{2}} \sigma_{t}^{(i)} \mathrm{d} W_{t},
$$

where $B^{(i)} \Perp W$. Throughout, we work with $i=1,2$. Note that $\rho^{(i)} \sigma_{t}^{(i)} \mathrm{d} B_{t}^{(i)}$ represents an idiosyncratic shock, while $\sqrt{1-\left[\rho^{(i)}\right]^{2}} \sigma_{t}^{(i)} \mathrm{d} W_{t}$ is a common factor.

The model for the diffusive volatility is specified as: $\sigma_{t}^{(i)}=\exp \left(\beta_{0}^{(i)}+\beta_{1}^{(i)} \varrho_{t}^{(i)}\right)$, where each of the $\varrho_{t}^{(i)}$ processes conform with Ornstein-Uhlenbeck dynamics: $\mathrm{d} \varrho_{t}^{(i)}=\alpha^{(i)} \varrho_{t}^{(i)} \mathrm{d} t+\mathrm{d} B_{t}^{(i)}$. This assumption means that the innovations 
Figure 1: Illustration of sampling schemes.

1)

2)

3)

Note. The figure illustrates how we design asynchronicity in the simulation study. A vertical dash (“"”) represents an observation of the noisy process $Y^{(1)}$, while a cross (“×”) is $Y^{(2)}$. A star (“*”) defines a common sampling point.

of $\rho^{(i)} \sigma_{t}^{(i)} \mathrm{d} B_{t}^{(i)}$ and $\mathrm{d} \sigma_{t}^{(i)}$ are perfectly correlated, while the covariation between $\mathrm{d} X_{t}^{(i)}$ and $\mathrm{d} \varrho_{t}^{(i)}$ is equal to $\rho^{(i)} \sigma_{t}^{(i)} \mathrm{d} t$. Finally, note that the model allows the two underlying price processes $X_{t}^{(1)}$ and $X_{t}^{(2)}$ to be correlated in the magnitude of $\sqrt{1-\left[\rho^{(1)}\right]^{2}} \sqrt{1-\left[\rho^{(2)}\right]^{2}}$.

We carry out our numerical experiments by using the following parametrization, assumed to be identical across the two volatility factors: $\left(a^{(i)}, \beta_{0}^{(i)}, \beta_{1}^{(i)}, \alpha^{(i)}, \rho^{(i)}\right)=(0.03,-5 / 16,1 / 8,-1 / 40,-0.3)$, so that $\beta_{0}^{(i)}=\left[\beta_{1}^{(i)}\right]^{2} /\left[2 \alpha^{(i)}\right]$. This choice of parameters implies that integrated volatility has been normalized, in the sense that $\mathbb{E}\left(\int_{0}^{1}\left[\sigma_{s}^{(i)}\right]^{2} \mathrm{~d} s\right)=1$.

We simulate 10,000 paths of this model over the interval $[0,1]$, which we partition into $N=23,400$ subintervals of equal length $1 / N$. In constructing noisy prices $Y^{(i)}$, we first generate a complete high-frequency record of $N$ equidistant observations of the efficient price $X^{(i)}$ using a standard Euler scheme. ${ }^{1}$ The initial values for the $\varrho_{t}^{(i)}$ processes at each simulation run are drawn randomly from their stationary distribution, which is $\varrho_{t}^{(i)} \sim N\left(0,\left[-2 \alpha^{(i)}\right]^{-1}\right)$.

Next, we add simulated microstructure noise $Y^{(i)}=X^{(i)}+\varepsilon^{(i)}$ by taking

$$
\varepsilon^{(i)} \mid\{\sigma, X\} \stackrel{\text { i.i.d }}{\sim} N\left(0, \omega^{2}\right) \quad \text { with } \quad \omega^{2}=\gamma^{2}\left(\frac{1}{N} \sum_{j=1}^{N} \sigma_{j / N}^{(i) 2}\right),
$$

where $\gamma$ is the so-called noise ratio parameter. This choice means that the variance of the noise process increases with the level of volatility of $X^{(i)}$, as documented by [5]. $\gamma$ takes the value 0.50 , which is a typical level of noise (e.g., [8]).

\footnotetext{
${ }^{1}$ Note that the Ornstein-Uhlenbeck process permits an exact discretization (see, e.g., [10]). We use that fact here to avoid committing errors in working out the discrete time distribution of $\mathrm{d} \varrho^{(i)}$ over time steps of size $1 / N$.
} 
Finally, in order to extract non-synchronous data from the complete synchronous high-frequency record, we proceed as follows (for reference, please see Figure 1). We consider three settings. In scenario 1), the sampling times of $Y^{(2)}$ form a subset of the observation grid of $Y^{(1)}$, but $Y^{(1)}$ is observed more frequently. Here, we use $n_{1}=3,900$ and $n_{2}=390$. In scenario 2), we take $n_{1}=n_{2}=3,900$, but shift the observation times of $Y^{(2)}$ to lie midway between those of $Y^{(1)}$. Finally, in scenario 3), we generate random observation times using two independent Poisson processes with intensity $\lambda_{1}$ and $\lambda_{2}$. Here $\lambda_{i}$ denotes the average waiting time for new data from process $Y^{(i)}$, so that a typical simulation will have $N / \lambda_{i}$ observations of $Y^{(i)}, i=1,2$. We set $\lambda_{1}=6$ and $\lambda_{2}=60$, which implies that the first asset is trading ten times faster than the second. Note that because we are simulating in discrete time, it is possible to see common points in the last setting, as depicted in the chart.

The choice of the remaining tuning parameters are the following: We use $\theta=0.15$ and set $k_{n}=\lceil\theta \sqrt{n}\rceil$, where $\lceil x\rceil$ is the ceil function. Moreover, to estimate the variance appearing in the CLT of $H Y_{k l}^{n}$, we use $V_{k l, k l}^{n, 1}$ defined in (4.2) with $\varpi=2$ and $\eta=7 / 12$.

Our initial numerical experimentations show that the raw estimator from Eq. (2.5) is slightly downward biased in finite samples. This is familiar from related estimators, such as [7], where an additional factor is applied to correct for the loss of summands induced by pre-averaging. Here, the problem is slightly more delicate, but nonetheless a relatively simple device can be used to adjust the estimator. In particular, we generate a bivariate Brownian motion $\left(B^{(1)}, B^{(2)}\right)$ with a known correlation $\rho$ (throughout, we use $\rho=1$ ), where the coordinates of these two processes are identical to $\left(Y^{(1)}, Y^{(2)}\right)$. We then estimate $R_{k l}^{n}=\mathbb{E}\left[H Y_{k l}^{n}\right]$ across 10,000 repetitions using the data from $B^{(1)}$ and $B^{(2)}$ and divide the original statistic $H Y_{k l}^{n}$ (based on data from $Y^{(1)}$ and $Y^{(2)}$ ) by $R_{k l}^{n} / \rho$. A similar procedure can be used to bias correct the estimator of variance.

\subsection{Simulation results}

In Table 1, we present the relative bias and root mean squared error of our pre-averaged Hayashi-Yoshida estimator. As a comparison, we also computed the modulated realised covariance (MRC) of [7] based on refresh time sampling. As the table reveals, both estimators are unbiased (after bias correction) in all three scenarios. $H Y_{22}^{n}$ does retain a slight bias in those scenarios, where $n_{2}$ is very small, but the bias is less than a percent. The rmse of $H Y^{n}$ is larger than what we observe for the MRC, when the estimation target is a variance component; this observation is in line with the theoretical comparison of Remark 3.5. This is particularly true for the slow-trading asset $Y^{(2)}$ in scenarios one and three. However, the rmse of $H Y_{12}^{n}$ is smaller than the rmse of the modulated realised covariance in all scenarios. As expected the estimator $H Y_{12}^{n}$ performs much better than MRC when the observation numbers $n_{1}$ and $n_{2}$ have a different order of magnitude (i.e. 
in scenarios one and three). It is explained by the fact that refresh time sampling essentially uses the slowest frequency. This highlights the advantages of our new estimator $H Y^{n}$.

Table 1: Relative bias and root mean squared error.

\begin{tabular}{lccccccc}
\hline & \multicolumn{3}{c}{$H Y^{n}$} & & \multicolumn{3}{c}{ MRC } \\
\cline { 2 - 4 } \cline { 6 - 7 } Target & $\Sigma_{11}$ & $\Sigma_{12}$ & $\Sigma_{22}$ & & $\Sigma_{11}$ & $\Sigma_{12}$ & $\Sigma_{22}$ \\
\hline Scenario 1 & 1.00 & 1.00 & 0.99 & & 1.00 & 1.00 & 1.00 \\
& $(0.19)$ & $(0.04)$ & $(0.27)$ & & $(0.16)$ & $(0.06)$ & $(0.14)$ \\
& & & & & & \\
Scenario 2 & 1.00 & 1.00 & 1.00 & & 1.00 & 1.00 & 1.00 \\
& $(0.14)$ & $(0.03)$ & $(0.13)$ & & $(0.07)$ & $(0.04)$ & $(0.08)$ \\
& & & & & & \\
Scenario 3 & 1.00 & 1.00 & 0.99 & & 1.00 & 1.00 & 1.00 \\
& $(0.21)$ & $(0.04)$ & $(0.31)$ & & $(0.17)$ & $(0.07)$ & $(0.15)$ \\
\hline
\end{tabular}

Note. We report the relative bias and rmse of the estimators included in the simulation study. The bias measure is equal to 1 for an unbiased estimator. The number reported in parenthesis is $1000 \times$ rmse.

Next, we turn to the accuracy of the asymptotic approximation, where we focus on estimation of integrated covariance, $\Sigma_{12}$. In Figure 2, we plot the simulated finite sample distribution of the standardized $H Y_{12}^{n}$ for the three setups considered here, where the variance of the estimator is accessed by $V_{12,12}^{n, 1}$ as described above. Although the approximation is not perfect, the goodness of the fit is surprisingly good taking the relatively small sample into account. Also, the ordering is as expected with the second scenario offering the best approximation to the standard normal (where $n_{1}=n_{2}=3,900$ ). Moreover, while the average number of observations is identical in scenario one and three, the randomness of the spacings in the latter setting slightly deteriorates the tracking of the standard normal.

\section{Proofs}

Let $C>0$ denote a generic constant which may change from line to line; we also write $C_{p}>0$ if a constant depends on an external parameter $p$. For the sake of simplicity we will sometimes keep the dependence of some quantities on certain parameters unreflected if things are clear from the context. Also some notations might have a different meaning in different subsections, e.g. the quantity $R_{n}(p)$ stands for a generic asymptotically negligible random variable in Sections 6.1.5-6.1.7. 
Figure 2: Accuracy of asymptotic approximation, estimation of $\Sigma_{12}$.

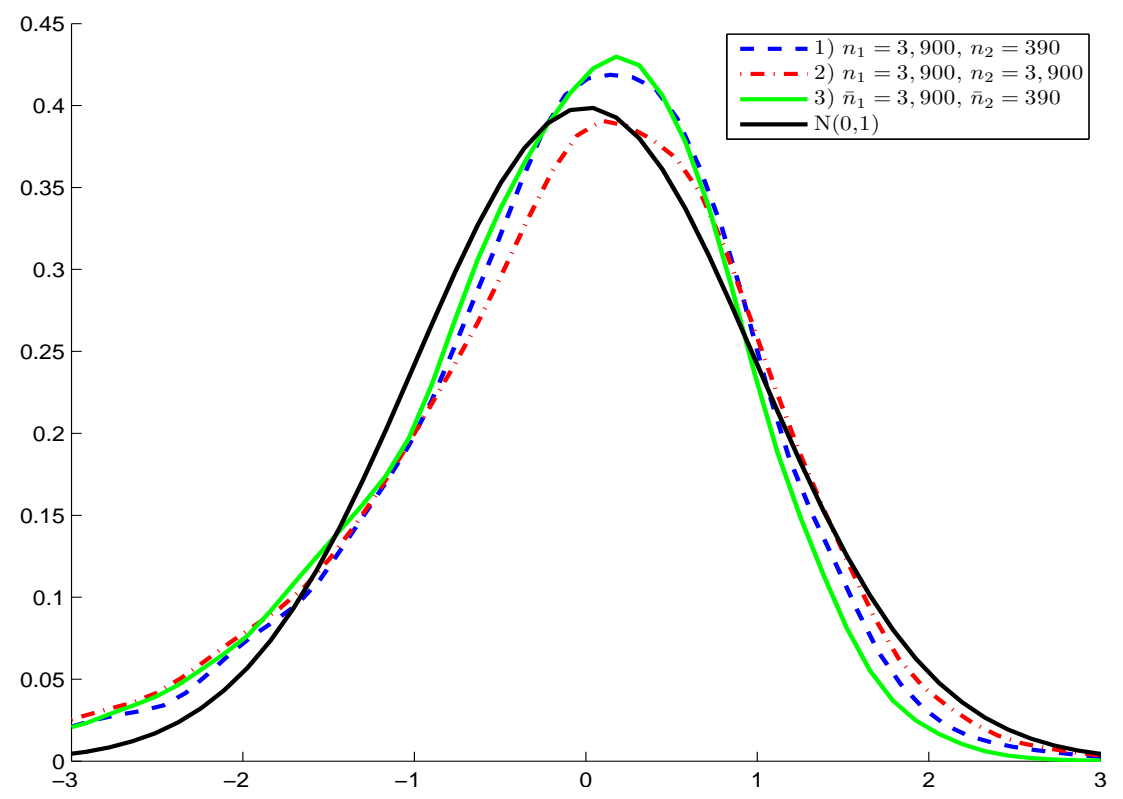

We remark that all our theoretical results (Theorems 3.1, 3.4, 4.1, 4.2, 4.3) are stable under localization, i.e. if they are valid for bounded coefficients then they remain valid for locally bounded coefficients. This means, since the processes $a$ and $\sigma$ are càglàd, thus locally bounded, we can assume without loss of generality:

- The processes $a$ and $\sigma$ are bounded in $(\omega, t)$.

See e.g. Section 3 in [2] for more details.

The second important step in all proofs is the approximation

$$
\bar{Y}_{t_{i}^{k}}^{k} \approx\left(\overline{\sigma_{t_{i}^{k}} W}\right)_{t_{i}^{k}}^{k}+\bar{\varepsilon}_{t_{i}^{k}}^{k}, \quad 1 \leq k \leq d,
$$

which means that we may pretend that $a=0$ identically and that the volatility $\sigma$ is constant over the small intervals $\left[t_{i}^{k}, t_{i+k_{n}}^{k}\right]$. Indeed, we will show that such an approximation does not affect any of our theoretical results.

Before we start proving our main results let us state some simple lemmas which concern the observation times $t_{i}^{k}$ and the pre-averaging quantities $\bar{Y}_{t_{i}^{k}}^{k}$. In what follows we use the decomposition

$$
X_{t}=X_{0}+D_{t}+N_{t}, \quad D_{t}=\int_{0}^{t} a_{s} d s, \quad N_{t}=\int_{0}^{t} \sigma_{s} d W_{s}
$$


We also decompose the statistic $H Y^{n}$ as

$$
H Y_{k l}^{n}=H Y_{k l}^{n}[X]+H Y_{k l}^{n}[X, \varepsilon]+H Y_{k l}^{n}[\varepsilon]
$$

with

$$
\begin{aligned}
H Y_{k l}^{n}[X] & =\frac{1}{\left(\psi k_{n}\right)^{2}} \sum_{i=0}^{n_{k}-k_{n}+1} \sum_{j=0}^{n_{l}-k_{n}+1} \bar{X}_{t_{i}^{k}}^{k} \bar{X}_{t_{j}^{l}}^{l} 1_{\left\{\left(t_{i}^{k}, t_{i+k_{n}}^{k}\right] \cap\left(t_{j}^{l}, t_{j+k_{n}}^{l}\right] \neq \emptyset\right\},}, \\
H Y_{k l}^{n}[X, \varepsilon] & =\frac{1}{\left(\psi k_{n}\right)^{2}} \sum_{i=0}^{n_{k}-k_{n}+1} \sum_{j=0}^{n_{l}-k_{n}+1}\left(\bar{X}_{t_{i}^{k}}^{k} \bar{\varepsilon}_{t_{j}^{l}}^{l}+\bar{\varepsilon}_{t_{i}^{k}}^{k} \bar{X}_{t_{j}^{l}}^{l}\right) 1_{\left\{\left(t_{i}^{k}, t_{i+k n}^{k}\right] \cap\left(t_{j}^{l}, t_{j+k_{n}}^{l}\right] \neq \emptyset\right\},}, \\
H Y_{k l}^{n}[\varepsilon] & =\frac{1}{\left(\psi k_{n}\right)^{2}} \sum_{i=0}^{n_{k}-k_{n}+1} \sum_{j=0}^{n_{l}-k_{n}+1} \bar{\varepsilon}_{t_{i}^{k}}^{k} \bar{\varepsilon}_{t_{j}^{l}}^{l} 1_{\left\{\left(t_{i}^{k}, t_{i+k_{n}}^{k}\right] \cap\left(t_{j}^{l}, t_{j+k_{n}}^{l}\right] \neq \emptyset\right\} .}
\end{aligned}
$$

Lemma 6.1 Under the Assumptions (T1)-(T3) we have for any $0 \leq a<b \leq 1$

$$
\sharp\left\{i \mid t_{i}^{k} \in[a, b]\right\} \leq C(b-a) n+1 \quad \forall 1 \leq k \leq d .
$$

Proof: To compute the cardinality of the above set we need to calculate $n_{k}\left(f_{k}(b)-f_{k}(a)\right)$, which is an upper bound for the number of points falling into $[a, b]$, up to adding one. The mean value theorem and conditions (T2), (T3) imply that

$$
n\left(f_{k}(b)-f_{k}(a)\right)=n_{k}\left(f_{k}\right)^{\prime}(\xi)(b-a) \leq C n(b-a)
$$

where $\xi$ is some point between $a$ and $b$.

The above lemma basically states that the amount of time points $t_{i}^{k}$ contained in $[a, b]$ is of the same order as in the equidistant case for all $k$.

Lemma 6.2 Under the Assumptions (T) and if $\mathbb{E}\left[\varepsilon^{8}\right]<\infty$ we obtain for $q=2,4,8$

$$
\mathbb{E}\left[\left|\bar{Y}_{t_{i}^{k}}^{k}\right|^{q}\right] \leq C n^{-q / 4}, \quad \mathbb{E}\left[\left|\bar{D}_{t_{i}^{k}}^{k}\right|^{q}\right] \leq C n^{-q / 2}, \quad \forall 1 \leq k \leq d, 1 \leq i \leq n_{k}
$$

Proof: These estimates are shown separately for $\bar{N}_{t_{i}^{k}}^{k}, \bar{D}_{t_{i}^{k}}^{k}$ and $\bar{\varepsilon}_{t_{i}^{k}}^{k}$. They are a simple consequence of the boundedness of the processes $a$ and $\sigma$, the Burkholder inequality and Lemma 6.1. See e.g. Section 5.4 from [15] for a detailed computation in the equidistant case. 


\subsection{Proof of Theorem 3.4}

Because the summands in the definition of the estimator $H Y^{n}$ are highly correlated, the main idea of the proof is to apply a similar method as for the proof of the central limit theorem for $m$-dependent data. Roughly speaking, we will collect all summands of $H Y^{n}$ in big and small blocks. The function of the small blocks is to ensure the (conditional) asymptotic independence of the big blocks, and their contribution will become negligible in the limit.

Let us start with the formal definition of big and small blocks. For some $p>0$, we set

$$
\begin{aligned}
& B_{z}(p)=\left[\frac{z(p+b) k_{n}}{n}, \frac{z(p+b) k_{n}+p k_{n}}{n}\right) \quad \text { (big blocks) } \\
& S_{z}(p)=\left[\frac{z(p+b) k_{n}+p k_{n}}{n}, \frac{(z+1)(p+b) k_{n}}{n}\right) \quad \text { (small blocks) }
\end{aligned}
$$

where $b$ is larger than $M \max _{1 \leq k \leq d}\left(m_{k}^{-1}\right)$ and $z=0, \ldots,\left[\frac{n}{(p+b) k_{n}}\right]-1$. The constant $b$ is chosen in this way to ensure that the quantities $\bar{Y}_{t_{i}^{k}}^{k}, \bar{Y}_{t_{j}^{l}}^{l}$ with $t_{i}^{k} \in B_{z}(p), t_{j}^{l} \in B_{z^{\prime}}(p)$ and $z \neq z^{\prime}$ do not use the same data, at least for $n$ large enough (see the proof of Lemma 6.1). This fact leads to the asymptotic conditional independence of the big blocks. The notion of big blocks comes from the fact that the length of $B_{z}(p)$ is always $p k_{n} / n$, where we later let $p \rightarrow \infty$, which is large compared to the length $b k_{n} / n$ of small blocks $S_{z}(p)$.

We will perform the proof in several steps. In a certain sense we will prove the statement in a reverse order. The road map of the proof is as follows:

(i) In Section 6.1.1 we will show a stable central limit theorem for the approximative quantities of the type (6.1), which are collected in big blocks $B_{z}(p)$. The corresponding stable limit is $L$ defined in Theorem 3.4.

(ii) In Section 6.1 .2 we will prove the asymptotic negligibility of the approximative quantities of the type (6.1) which are collected in small blocks $S_{z}(p)$.

(iii) Sections 6.1.3-6.1.7 are devoted to the justification of the approximation in (6.1): Sections 6.1.3-6.1.5 deal with the diffusion part (the most involved part), Section 6.1.6 treats the mixed part and Section 6.1.7 is devoted to the noise part.

(iv) Section 6.1.4 provides a useful decomposition for the diffusion part, which shows that our statistic $H Y^{n}$ is asymptotically unbiased. 


\subsubsection{The central limit theorem for the big blocks}

Whenever $t_{i}^{k} \in A_{z}(p), t_{j}^{l} \in A_{z^{\prime}}(p)$ for $A=B$ or $A=S$ (see (6.4)), we set

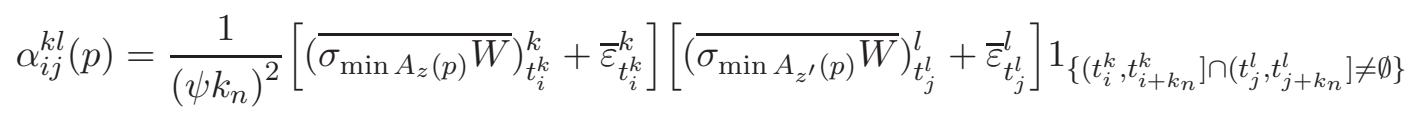

Here we follow the same approximation as in (6.1), except the volatility process is now frozen in the beginning of the block $A_{z}(p)$ resp. $A_{z^{\prime}}(p)$. We define $M_{n}^{k l}(p)=\sum_{z} \zeta_{z n}^{k l}(p)$ with

$$
\zeta_{z n}^{k l}(p)=n^{1 / 4} \sum_{t_{i}^{k}, t_{j}^{l} \in B_{z}(p)}\left(\alpha_{i j}^{k l}(p)-\mathbb{E}\left[\alpha_{i j}^{k l}(p) \mid \mathcal{F}_{\min B_{z}(p)}\right]\right) .
$$

As $M_{n}^{k l}(p)$ is a quadratic form of $Y=X+\varepsilon$, we have a straightforward decomposition

$$
M_{n}^{k l}(p)=M_{n}^{k l}(X, p)+M_{n}^{k l}(X, \varepsilon, p)+M_{n}^{k l}(\varepsilon, p),
$$

where $M_{n}^{k l}(X, p)$ denotes the diffusion part of $M_{n}^{k l}(p), M_{n}^{k l}(\varepsilon, p)$ stands for the noise part of $M_{n}^{k l}(p)$ and $M_{n}^{k l}(X, \varepsilon, p)$ is the mixed part of $M_{n}^{k l}(p)$, which will be used in the following sections. In these we will show that the quantities $M_{n}(p)$ and $L^{n}=n^{1 / 4}\left(H Y^{n}-[X]\right)$ are asymptotically equivalent, i.e.

$$
\lim _{p \rightarrow \infty} \limsup _{n \rightarrow \infty} \mathbb{P}\left(\left|M_{n}(p)-L^{n}\right|>\delta\right)=0
$$

for all $\delta>0$. Thus, it is sufficient to prove the following result which completes this section.

Theorem 6.3 Assume that the conditions of Theorem 3.4 hold. Then we obtain that

$$
M_{n}(p) \stackrel{d_{s t}}{\longrightarrow} M(p)=M N\left(0, V_{p}\right) \quad \text { as } n \rightarrow \infty
$$

for a certain conditional covariance matrix $V_{p}$. Furthermore, when $p \rightarrow \infty$ we deduce that $V_{p} \stackrel{\mathbb{P}}{\longrightarrow} V$, thus

$$
M(p) \stackrel{\mathbb{P}}{\longrightarrow} L=M N(0, V),
$$

where the random variables $V$ and $L$ are defined in Theorem 3.4.

Proof: By Theorem IX.7.28 from [17] it is sufficient to show that $\left(1 \leq k, l, k^{\prime}, l^{\prime} \leq d\right)$

(i) $\sum_{z} \mathbb{E}\left[\zeta_{z n}^{k l}(p) \zeta_{z n}^{k^{\prime} l^{\prime}}(p) \mid \mathcal{F}_{\min B_{z}(p)}\right] \stackrel{\mathbb{P}}{\longrightarrow} V_{p}^{k l, k^{\prime} l^{\prime}}$ 
(ii) $\sum_{z} \mathbb{E}\left[\zeta_{z n}^{k l}(p)\left(W_{\max B_{z}(p)}^{k^{\prime}}-W_{\min B_{z}(p)}^{k^{\prime}}\right) \mid \mathcal{F}_{\min B_{z}(p)}\right] \stackrel{\mathbb{P}}{\longrightarrow} 0$,

(iii) $\sum_{z} \mathbb{E}\left[\left|\zeta_{z n}^{k l}(p)\right|^{4}\right] \rightarrow 0$

(iv) $\sum_{z} \mathbb{E}\left[\zeta_{z n}^{k l}(p)\left(N_{\max B_{z}(p)}-N_{\min B_{z}(p)}\right) \mid \mathcal{F}_{\min B_{z}(p)}\right] \stackrel{\mathbb{P}}{\longrightarrow} 0$ for all bounded martingales $N$ with $\langle N, W\rangle=0$,

to conclude the stable convergence $M_{n}(p) \stackrel{d_{s t}}{\longrightarrow} M(p)$ as $n \rightarrow \infty$. The statement (i) is proved in the Appendix. To show (ii) we remark that the increments of $W$ involved in $\zeta_{z n}^{k l}$ are independent of $\mathcal{F}_{\min B_{z}(p)}$. On the other hand, the quantity $\zeta_{z n}^{k l}(p)\left(W_{\max B_{z}(p)}^{k^{\prime}}-W_{\min B_{z}(p)}^{k^{\prime}}\right)$ is an odd function of $W$ and $(W, \varepsilon) \stackrel{d}{=}(-W, \varepsilon)$ since $W, \varepsilon$ are independent, which implies that

$$
\mathbb{E}\left[\zeta_{z n}^{k l}(p)\left(W_{\max B_{z}(p)}^{k^{\prime}}-W_{\min B_{z}(p)}^{k^{\prime}}\right) \mid \mathcal{F}_{\min B_{z}(p)}\right]=0 .
$$

Next, to show (iii) we observe that for fixed $p$ the number of summands involved in the definition of $\zeta_{z n}^{k l}(p)$ is $O\left(k_{n}^{2}\right)$. Due to Lemma 6.2 and since $z=0, \ldots,\left[\frac{n}{(p+b) k_{n}}\right]-1$ we immediately deduce that

$$
\sum_{z} \mathbb{E}\left[\left|\zeta_{z n}^{k l}(p)\right|^{4}\right] \leq C_{p} \frac{n}{(p+b) k_{n}} n k_{n}^{8}\left(k_{n}\right)^{-8} n^{-2} \leq \frac{C_{p}}{k_{n}} \rightarrow 0 .
$$

Part (iv) is shown in [15] for an analogous situation (see Lemma 5.7 therein). This completes the proof of the first statement of Theorem 6.3. The second statement is again proved in the Appendix. 


\subsubsection{Negligibility of the small blocks}

In this section we still consider the approximative quantities $\alpha_{i j}^{k l}(p)$ from (6.5) and show that the term $\widetilde{M}_{n}^{k l}(p)=$ $\sum_{z} \widetilde{\zeta}_{z n}^{k l}(p)$ with $\widetilde{\zeta}_{z n}^{k l}(p)=\sum_{i=1}^{5} \widetilde{\zeta}_{z n}^{k l}(i, p)$ given as

$$
\begin{aligned}
& \widetilde{\zeta}_{z n}^{k l}(1, p)=n^{1 / 4} \sum_{t_{i}^{k}, t_{j}^{l} \in S_{z}(p)}\left(\alpha_{i j}^{k l}(p)-\mathbb{E}\left[\alpha_{i j}^{k l}(p) \mid \mathcal{F}_{\left.\min S_{z}(p)\right]}\right]\right) \\
& \widetilde{\zeta}_{z n}^{k l}(2, p)=n^{1 / 4} \sum_{t_{i}^{k} \in B_{z-1}(p), t_{j}^{l} \in S_{z}(p)}\left(\alpha_{i j}^{k l}(p)-\mathbb{E}\left[\alpha_{i j}^{k l}(p) \mid \mathcal{F}_{\min B_{z-1}(p)}\right]\right) \\
& \widetilde{\zeta}_{z n}^{k l}(3, p)=n^{1 / 4} \sum_{t_{i}^{k} \in B_{z+1}(p), t_{j}^{l} \in S_{z}(p)}\left(\alpha_{i j}^{k l}(p)-\mathbb{E}\left[\alpha_{i j}^{k l}(p) \mid \mathcal{F}_{\left.\min S_{z}(p)\right]}\right)\right. \\
& \widetilde{\zeta}_{z n}^{k l}(4, p)=n^{1 / 4} \sum_{t_{j}^{l} \in B_{z-1}(p), t_{i}^{k} \in S_{z}(p)}\left(\alpha_{i j}^{k l}(p)-\mathbb{E}\left[\alpha_{i j}^{k l}(p) \mid \mathcal{F}_{\min B_{z-1}(p)}\right]\right) \\
& \widetilde{\zeta}_{z n}^{k l}(5, p)=n^{1 / 4} \sum_{t_{j}^{l} \in B_{z+1}(p), t_{i}^{k} \in S_{z}(p)}\left(\alpha_{i j}^{k l}(p)-\mathbb{E}\left[\alpha_{i j}^{k l}(p) \mid \mathcal{F}_{\min S_{z}(p)}\right]\right),
\end{aligned}
$$

is negligible in the sense of (6.7). This representation holds for $p>b$ (see (6.4) for the definition of the constant $b$ ), which we assume without loss of generality. As in (6.6), we have the decomposition

$$
\widetilde{M}_{n}^{k l}(p)=\widetilde{M}_{n}^{k l}(X, p)+\widetilde{M}_{n}^{k l}(X, \varepsilon, p)+\widetilde{M}_{n}^{k l}(\varepsilon, p),
$$

into the $X$-part, the mixed part and the $\varepsilon$-part, which will be used in the following sections. Let us consider the term $\sum_{z} \widetilde{\zeta}_{z n}^{k l}(1, p)$. First of all, we remark that the summands $\widetilde{\zeta}_{z n}^{k l}(1, p)$ are uncorrelated (as $z$ runs) and the number of summands is of order $n /\left(p k_{n}\right)$. Furthermore, there are $O\left(k_{n}^{2}\right)$ summands in the definition of $\widetilde{\zeta}_{z n}^{k l}(1, p)$. Thus, we conclude from Lemma 6.2 that

$$
\mathbb{E}\left(\left|\sum_{z} \widetilde{\zeta}_{z n}^{k l}(1, p)\right|^{2}\right)=\sum_{z} \mathbb{E}\left[\left|\widetilde{\zeta}_{z n}^{k l}(1, p)\right|^{2}\right] \leq \frac{C}{p}
$$

Hence, we obtain

$$
\lim _{p \rightarrow \infty} \limsup _{n \rightarrow \infty} \mathbb{P}\left(\left|\sum_{z} \widetilde{\zeta}_{z n}^{k l}(1, p)\right|>\delta\right)=0
$$

for all $\delta>0$. The same assertion holds for $\widetilde{M}_{n}^{k l}(p)$, as counting the number of non-zero $\alpha_{i j}^{k l}(p)$ for $t_{i}^{k}$ and $t_{j}^{l}$ from disjoint blocks shows that the upper bound in (6.9) is valid for $\widetilde{\zeta}_{z n}^{k l}(q, p)$ as well, $q=2, \ldots, 5$. 


\subsubsection{The approximation of the diffusion part I}

We start with the decomposition of the diffusion part of the estimator $H Y^{n}$. Set $H Y_{k l}^{n}[X]=H Y_{k l}^{n}[D]+H Y_{k l}^{n}[D, N]+$ $H Y_{k l}^{n}[N]$ with

$$
\begin{aligned}
H Y_{k l}^{n}[D] & =\frac{1}{\left(\psi k_{n}\right)^{2}} \sum_{i=0}^{n_{k}-k_{n}+1} \sum_{j=0}^{n_{l}-k_{n}+1} \bar{D}_{t_{i}^{k}}^{k} \bar{D}_{t_{j}^{l}}^{l} 1_{\left\{\left(t_{i}^{k}, t_{i+k_{n}}^{k}\right] \cap\left(t_{j}^{l}, t_{j+k_{n}}^{l}\right] \neq \emptyset\right\},} \\
H Y_{k l}^{n}[D, N] & =\frac{1}{\left(\psi k_{n}\right)^{2}} \sum_{i=0}^{n_{k}-k_{n}+1} \sum_{j=0}^{n_{l}-k_{n}+1}\left(\bar{D}_{t_{i}^{k}}^{k} \bar{N}_{t_{j}^{l}}^{l}+\bar{N}_{t_{i}^{k}}^{k} \bar{D}_{t_{j}^{l}}^{l}\right) 1_{\left\{\left(t_{i}^{k}, t_{i+k_{n}}^{k}\right] \cap\left(t_{j}^{l}, t_{j+k_{n}}^{l}\right] \neq \emptyset\right\},}, \\
H Y_{k l}^{n}[N] & =\frac{1}{\left(\psi k_{n}\right)^{2}} \sum_{i=0}^{n_{k}-k_{n}+1} \sum_{j=0}^{n_{l}-k_{n}+1} \bar{N}_{t_{i}^{k}}^{k} \bar{N}_{t_{j}^{l}}^{l} 1_{\left\{\left(t_{i}^{k}, t_{i+k_{n}}^{k}\right] \cap\left(t_{j}^{l}, t_{j+k_{n}}^{l}\right] \neq \emptyset\right\}},
\end{aligned}
$$

where the processes $D$ and $N$ are given in (6.2). In this section we will show that drift part $D$ of $X$ does not influence the central limit theorem, i.e.

$$
H Y_{k l}^{n}[D]=o_{\mathbb{P}}\left(n^{-1 / 4}\right), \quad H Y_{k l}^{n}[D, N]=o_{\mathbb{P}}\left(n^{-1 / 4}\right) .
$$

We start with the term $H Y_{k l}^{n}[D]$. Note that $H Y_{k l}^{n}[D]$ contains $O\left(n k_{n}\right)$ non-zero summands (due to Lemma 6.1). Lemma 6.2 and the Cauchy-Schwarz inequality imply that each summand satisfies $\mathbb{E}\left[\left|\bar{D}_{t_{i}^{k}}^{k} \bar{D}_{t_{j}^{l}}^{l}\right|\right] \leq C n^{-1}$. Thus, $\mathbb{E}\left[\left|H Y_{k l}^{n}[D]\right|\right] \leq$ $C n^{-1 / 2}$, which implies $H Y_{k l}^{n}[D]=o \mathbb{P}\left(n^{-1 / 4}\right)$.

The treatment of $H Y_{k l}^{n}[D, N]$ is a bit more delicate. We set

$$
\xi_{i j}^{n}=\bar{D}_{t_{i}^{k}}^{k} \bar{N}_{t_{j}^{l}}^{l}+\bar{N}_{t_{i}^{k}}^{k} \bar{D}_{t_{j}^{l}}^{l}
$$

and define

$$
\widetilde{\xi}_{i j}^{n}=a_{t_{i}^{k} \wedge t_{j}^{l}}\left(\overline{\mathrm{id}}_{t_{i}^{k}}^{k} \bar{N}_{t_{j}^{l}}^{l}+\bar{N}_{t_{i}^{k}}^{k} \overline{\mathrm{id}}_{t_{j}^{l}}^{l}\right),
$$

where id denotes the identity function on $\mathbb{R}$. The latter approximates $\xi_{i j}^{n}$ by freezing the process $a$ in a small time interval. Let us set

$$
\widetilde{H Y}_{k l}^{n}[D, N]=\frac{1}{\left(\psi k_{n}\right)^{2}} \sum_{i=0}^{n_{k}-k_{n}+1} \sum_{j=0}^{n_{l}-k_{n}+1} \widetilde{\xi}_{i j}^{n} 1_{\left\{\left(t_{i}^{k}, t_{i+k_{n}}^{k}\right] \cap\left(t_{j}^{l}, t_{j+k_{n}}^{l}\right] \neq \emptyset\right\}}
$$

We first show that $\widetilde{H Y}_{k l}^{n}[D, N]=o_{\mathbb{P}}\left(n^{-1 / 4}\right)$. Observe that

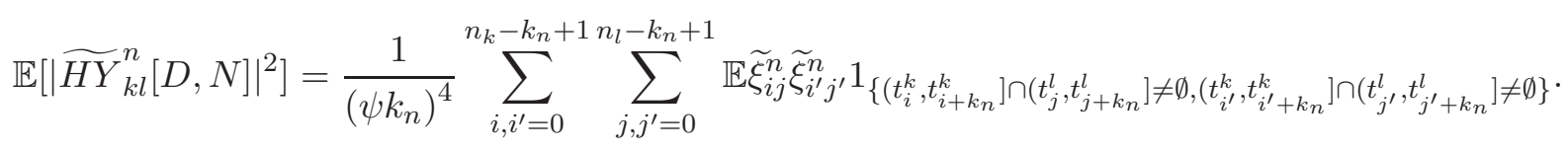

Due to Lemma 6.1 the above sum contains $O\left(n k_{n}^{3}\right)$ non-zero summands, because the $\widetilde{\xi}_{i j}^{n}$,s are martingale differences. Moreover, we have $\mathbb{E}\left[\left|\widetilde{\xi}_{i j}^{n}\right|^{2}\right] \leq C n^{-3 / 2}$ due to Lemma 6.2. Thus, we conclude $\mathbb{E}\left[\left|\widetilde{H Y}_{k l}^{n}[D, N]\right|^{2}\right] \leq C n^{-1}$, which 
implies that $\widetilde{H Y}_{k l}^{n}[D, N]=o \mathbb{P}\left(n^{-1 / 4}\right)$. In a second step we show that $H Y_{k l}^{n}[D, N]-\widetilde{H Y}_{k l}^{n}[D, N]=o \mathbb{P}\left(n^{-1 / 4}\right)$. For this purpose, for any càglàd bounded multivariate process $f$, we denote by $N_{\delta}^{f}(t)$ the number of jumps of $f$ bigger than $\delta>0$ before time $t$. Furthermore, we define

$$
m_{\eta, \delta}(f)=\sup \left\{\left\|f_{s}-f_{t}\right\|: t \leq s \leq(t+\eta) \wedge 1, N_{\delta}^{f}(s)-N_{\delta}^{f}(t)=0\right\} .
$$

Roughly speaking, $m_{\eta, \delta}(f)$ is a modulus of continuity of $f$ on intervals of at most length $\eta$, which do not contain jumps bigger than $\delta$. For $f$ as above, we obviously have $\lim _{\delta \rightarrow 0} \lim _{\sup _{\eta \rightarrow 0}} m_{\eta, \delta}(f)=0, \mathbb{P}-$ a.s. Observe that

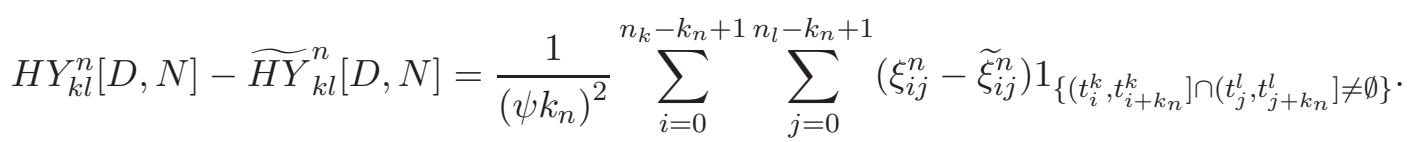

As we mentioned the above sum contains $O\left(n k_{n}\right)$ summands. We have

$$
\left|\bar{D}_{t_{i}^{k}}^{k}-a_{t_{i}^{k} \wedge t_{j}^{l}} \overline{\mathrm{i}}_{t_{i}^{k}}^{k}\right| \leq \sum_{h=1}^{k_{n}-1}\left|g\left(\frac{h}{k_{n}}\right)\right| \int_{t_{i+h-1}^{k}}^{t_{i+h}^{k}}\left\|a_{s}-a_{t_{i}^{k} \wedge t_{j}^{l}}\right\| d s .
$$

The right-hand side of the above inequality is bounded since the process $a$ is bounded by $C n^{-1 / 2}$. Consequently, distinguishing between the two situations, where $a$ has or does not have jumps bigger than $\delta$ in the interval $\left[t_{i+h-1}^{k}, t_{i+h}^{k}\right]$, we obtain the inequality

$$
\sum_{h=1}^{k_{n}-1}\left|g\left(\frac{h}{k_{n}}\right)\right| \int_{t_{i+h-1}^{k}}^{t_{i+h}^{k}}\left\|a_{s}-a_{t_{i}^{k} \wedge t_{j}^{l}}\right\| d s \leq C n^{-1 / 2}\left(m_{C k_{n} / n, \delta}(a)+\left(\left\{N_{\delta}^{a}\left(t_{i+k_{n}}^{k}\right)-N_{\delta}^{a}\left(t_{i}^{k} \wedge t_{j}^{l}\right)\right\} \wedge 1\right)\right) .
$$

Using Lemma 6.2 and Cauchy-Schwarz inequality we deduce that

$$
n^{1 / 4} \mathbb{E}\left[\left|H Y_{k l}^{n}[D, N]-\widetilde{H Y}_{k l}^{n}[D, N]\right|\right] \leq C \mathbb{E}\left[m_{C k_{n} / n, \delta}^{2}(a)+\left(\frac{N_{\delta}^{a}(1)}{n} \wedge 1\right)^{2}\right]^{1 / 2}
$$

Due to the dominated convergence theorem we conclude that

$$
\operatorname{lims}_{\delta \rightarrow 0} \limsup _{n \rightarrow \infty} \mathbb{E}\left[m_{C k_{n} / n, \delta}^{2}(a)+\left(\frac{N_{\delta}^{a}(1)}{n} \wedge 1\right)^{2}\right]=0 .
$$

Thus $H Y_{k l}^{n}[D, N]-\widetilde{H Y}_{k l}^{n}[D, N]=o_{\mathbb{P}}\left(n^{-1 / 4}\right)$. Summarizing all results of this section we get

$$
n^{1 / 4}\left(H Y_{k l}^{n}[X]-H Y_{k l}^{n}[N]\right)=o_{\mathbb{P}}(1)
$$

meaning that the martingale part $N$ is the dominating term in the decomposition of $H Y_{k l}^{n}[X]$. 


\subsubsection{A decomposition for the martingale part}

Having proved in the previous section that $H Y^{n}[X]$ can be replaced by $H Y^{n}[N]$ without affecting the limit, we proceed with a further decomposition of $H Y^{n}[N]$. In this section we will show that $H Y^{n}[N]$ is essentially an unbiased estimator of $\int_{0}^{1} \Sigma_{s} d s$. Recall that

$$
H Y_{k l}^{n}[N]=\frac{1}{\left(\psi k_{n}\right)^{2}} \sum_{i=0}^{n_{k}-k_{n}+1} \sum_{j=0}^{n_{l}-k_{n}+1} \bar{N}_{t_{i}^{k}}^{k} \bar{N}_{t_{j}^{l}}^{l} 1_{\left\{\left(t_{i}^{k}, t_{i+k_{n}}^{k}\right] \cap\left(t_{j}^{l}, t_{j+k_{n}}^{l}\right] \neq \emptyset\right\}}
$$

By definition we have

$$
\bar{N}_{t_{i}^{k}}^{k} \bar{N}_{t_{j}^{l}}^{l}=\sum_{h, h^{\prime}=1}^{k_{n}-1} g\left(\frac{h}{k_{n}}\right) g\left(\frac{h^{\prime}}{k_{n}}\right) \Delta_{t_{i+h}^{k}} N^{k} \Delta_{t_{j+h^{\prime}}^{l}} N^{l}\left(1_{E_{i j}^{h h^{\prime}}}+1_{\left(E_{i j}^{h h^{\prime}}\right)^{c}}\right)
$$

with

$$
E_{i j}^{h h^{\prime}}=\left\{\left(t_{i+h-1}^{k}, t_{i+h}^{k}\right] \cap\left(t_{j+h^{\prime}-1}^{l}, t_{j+h}^{l}\right] \neq \emptyset\right\} .
$$

Now, we will write the above quantity as a sum of martingale differences plus bias. For this purpose we need some additional notations. We decompose $E_{i j}^{h h^{\prime}}=\cup_{r=1}^{4} E_{i j}^{h h^{\prime}}(r)$ with

$$
\begin{aligned}
& E_{i j}^{h h^{\prime}}(1)=\left\{(i, j),\left(h, h^{\prime}\right) \mid t_{j+h^{\prime}-1}^{l} \geq t_{i+h-1}^{k}, \quad t_{j+h^{\prime}}^{l} \geq t_{i+h}^{k}\right\} \cap E_{i j}^{h h^{\prime}} \\
& E_{i j}^{h h^{\prime}}(2)=\left\{(i, j),\left(h, h^{\prime}\right) \mid t_{j+h^{\prime}-1}^{l} \geq t_{i+h-1}^{k}, \quad t_{j+h^{\prime}}^{l}<t_{i+h}^{k}\right\} \cap E_{i j}^{h h^{\prime}} \\
& E_{i j}^{h h^{\prime}}(3)=\left\{(i, j),\left(h, h^{\prime}\right) \mid t_{j+h^{\prime}-1}^{l}<t_{i+h-1}^{k}, \quad t_{j+h^{\prime}}^{l}<t_{i+h}^{k}\right\} \cap E_{i j}^{h h^{\prime}} \\
& E_{i j}^{h h^{\prime}}(4)=\left\{(i, j),\left(h, h^{\prime}\right) \mid t_{j+h^{\prime}-1}^{l}<t_{i+h-1}^{k}, \quad t_{j+h^{\prime}}^{l} \geq t_{i+h}^{k}\right\} \cap E_{i j}^{h h^{\prime}}
\end{aligned}
$$

On $E_{i j}^{h h^{\prime}}(1)$ we deduce by Itô formula:

$$
\begin{aligned}
& \Delta_{t_{i+h}^{k}} N^{k} \Delta_{t_{j+h^{\prime}}^{l}} N^{l}=\left(N_{t_{j+h^{\prime}-1}^{l}}^{k}-N_{t_{i+h-1}^{k}}^{k}\right) \Delta_{t_{j+h^{\prime}}^{l}} N^{l}+\left(N_{t_{i+h}^{k}}^{k}-N_{t_{j+h^{\prime}-1}^{l}}^{k}\right)\left(N_{t_{j+h^{\prime}}^{l}}^{l}-N_{t_{i+h}^{k}}^{l}\right) \\
& +\int_{t_{j+h^{\prime}-1}^{l}}^{t_{i+h}^{k}}\left(N_{s}^{k}-N_{t_{j+h^{\prime}-1}^{l}}^{k}\right) d N_{s}^{l}+\int_{t_{j+h^{\prime}-1}^{l}}^{t_{i+h}^{k}}\left(N_{s}^{l}-N_{t_{j+h^{\prime}-1}^{l}}^{l}\right) d N_{s}^{k}+\int_{t_{j+h^{\prime}-1}^{l} t_{i+h}^{k}} \Sigma_{s}^{k l} d s \\
& =\sum_{r=1}^{5} \mu_{i j}^{h h^{\prime}}(1, r),
\end{aligned}
$$

and similar decompositions are obtained on $E_{i j}^{h h^{\prime}}(q), q=2,3,4$, and we denote them by $\sum_{r=1}^{5} \mu_{i j}^{h h^{\prime}}(q, r)$. Notice that all terms $\mu_{i j}^{h h^{\prime}}(q, r)$ are martingale differences for $1 \leq q, r \leq 4$, while $\mu_{i j}^{h h^{\prime}}(q, 5)$ gives the bias for all $1 \leq q \leq 4$. We define

$$
\mu_{i j}(q, r)=\sum_{h, h^{\prime}=1}^{k_{n}-1} g\left(\frac{h}{k_{n}}\right) g\left(\frac{h^{\prime}}{k_{n}}\right) \mu_{i j}^{h h^{\prime}}(q, r) 1_{E_{i j}^{h h^{\prime}}(q)}
$$


for $1 \leq q \leq 4,1 \leq r \leq 5$. Now, a simple reordering shows that

$$
\begin{aligned}
& \frac{1}{\left(\psi k_{n}\right)^{2}} \sum_{i, j}\left(\sum_{q=1}^{4} \mu_{i j}(q, 5)\right) 1_{\left\{\left(t_{i}^{k}, t_{i+k_{n}}^{k}\right] \cap\left(t_{j}^{l}, t_{j+k_{n}}^{l}\right] \neq \emptyset\right\}}=\frac{\sum_{h, h^{\prime}=1}^{k_{n}-1} g\left(\frac{h}{k_{n}}\right) g\left(\frac{h^{\prime}}{k_{n}}\right)}{\left(\psi k_{n}\right)^{2}} \int_{0}^{1} \Sigma_{s}^{k l} d s+o_{\mathbb{P}}\left(n^{-1 / 4}\right) \\
& =\int_{0}^{1} \Sigma_{s}^{k l} d s+o_{\mathbb{P}}\left(n^{-1 / 4}\right),
\end{aligned}
$$

where the error in the first identity is due to border effects, and the second identity uses $\psi=\int_{0}^{1} g(x) d x$.

Thus, we conclude that

$$
n^{1 / 4}\left(H Y_{k l}^{n}[N]-\int_{0}^{1} \Sigma_{s}^{k l} d s\right)=\frac{n^{1 / 4}}{\left(\psi k_{n}\right)^{2}} \sum_{i=0}^{n_{k}-k_{n}+1} \sum_{j=0}^{n_{l}-k_{n}+1} \eta_{i j}^{k l} 1_{\left\{\left(t_{i}^{k}, t_{i+k_{n}}^{k}\right] \cap\left(t_{j}^{l}, t_{j+k_{n}}^{l}\right] \neq \emptyset\right\}}+o_{\mathbb{P}}(1),
$$

where

$$
\begin{gathered}
\eta_{i j}^{k l}=\bar{\mu}_{i j}+\sum_{q, r=1}^{4} \mu_{i j}(q, r), \\
\bar{\mu}_{i j}=\sum_{h, h^{\prime}=1}^{k_{n}-1} g\left(\frac{h}{k_{n}}\right) g\left(\frac{h^{\prime}}{k_{n}}\right) \Delta_{t_{i+h}^{k}} N^{k} \Delta_{t_{j+h^{\prime}}^{l}} N^{l} 1_{\left(E_{i j}^{h h^{\prime}}\right)^{c} .}
\end{gathered}
$$

We remark again all terms $\eta_{i j}^{k l}$ are now sums of martingale differences.

\subsubsection{The approximation of the diffusion part II}

In this section we will justify the approximation

$$
n^{1 / 4}\left(H Y_{k l}^{n}[N]-\int_{0}^{1} \Sigma_{s}^{k l} d s\right)=M_{n}^{k l}(X, p)+\widetilde{M}_{n}^{k l}(X, p)+R_{n}^{k l}(p),
$$

where $M_{n}(X, p)$ and $\widetilde{M}_{n}(X, p)$ are defined by (6.6) and (6.8) respectively, for some $R_{n}^{k l}(p)$ with

$$
\lim _{p \rightarrow \infty} \limsup _{n \rightarrow \infty} \mathbb{P}\left(\left|R_{n}^{k l}(p)\right|>\delta\right)=0
$$

for all $\delta>0$. This means that the diffusion part $n^{1 / 4}\left(H Y_{k l}^{n}[N]-\int_{0}^{1} \Sigma_{s}^{k l} d s\right)$ of our statistic is asymptotically equivalent to the sum of the diffusion parts of big and small blocks. Recalling the estimate (6.15) from the previous section, it is easy to show

$$
\begin{aligned}
& R_{n}^{k l}(p)=n^{1 / 4}\left(H Y_{k l}^{n}[N]-\int_{0}^{1} \Sigma_{s}^{k l} d s\right)-M_{n}^{k l}(X, p)-\widetilde{M}_{n}^{k l}(X, p) \\
& =\frac{n^{1 / 4}}{\left(\psi k_{n}\right)^{2}} \sum_{i, j}\left(\eta_{i j}^{k l}-\widetilde{\eta}_{i j}^{k l}\right) 1_{\left\{\left(t_{i}^{k}, t_{i+k_{n}}^{k}\right] \cap\left(t_{j}^{l}, t_{j+k_{n}}^{l}\right] \neq \emptyset\right\}}+o_{\mathbb{P}}(1),
\end{aligned}
$$


where $\widetilde{\eta}_{i j}^{k l}$ is defined in the same way as $\eta_{i j}^{k l}$ (see (6.16)) except the process $N^{k}$ (resp. $\left.N^{l}\right)$ is replaced by $\left(\sigma_{\min } A_{z}(p) W\right)^{k}$ (resp. $\left.\left(\sigma_{\min A_{z^{\prime}}(p)} W\right)^{l}\right)$ when $t_{i}^{k} \in A_{z}(p)$ for some $z$ (resp. $t_{j}^{k} \in A_{z^{\prime}}(p)$ for some $z^{\prime}$ ) and $A=B$ or $A=S$. Note that the only difference compared to proving (6.15) lies in the fact that $M_{n}^{k l}(X, p)+\widetilde{M}_{n}^{k l}(X, p)$ is unbiased by construction.

Recall that the quantity $\eta_{i j}^{k l}$ (resp. $\widetilde{\eta}_{i j}^{k l}$ ) consists of 17 summands. Hence, we have the decomposition $R_{n}^{k l}(p)=$ $\sum_{r=1}^{17} R_{n}^{k l}(p, r)$. As an example we will only consider the treatment of the first summand, i.e.

$$
R_{n}^{k l}(p, 1)=\frac{n^{1 / 4}}{\left(\psi k_{n}\right)^{2}} \sum_{i, j}\left(\bar{\mu}_{i j}-\widetilde{\bar{\mu}}_{i j}\right) 1_{\left\{\left(t_{i}^{k}, t_{i+k_{n}}^{k}\right] \cap\left(t_{j}^{l}, t_{j+k_{n}}^{l}\right] \neq \emptyset\right\}},
$$

where $\bar{\mu}_{i j}$ is defined by (6.17). We conclude that

$$
\begin{aligned}
& \mathbb{E}\left[\left|\bar{\mu}_{i j}-\widetilde{\bar{\mu}}_{i j}\right|^{2}\right]=\mathbb{E}\left[\sum_{h, h^{\prime}, q, q^{\prime}} g\left(\frac{h}{k_{n}}\right) g\left(\frac{h^{\prime}}{k_{n}}\right) g\left(\frac{q}{k_{n}}\right) g\left(\frac{q^{\prime}}{k_{n}}\right) \Delta_{t_{i+h}^{k}}\left(N-\sigma_{\min A_{z}(p)} W\right)^{k}\right. \\
& \times \Delta_{t_{j+h^{\prime}}^{l}}\left(N-\sigma_{\min A_{z^{\prime}}(p)} W\right)^{l} \Delta_{t_{i+q}^{k}}\left(N-\sigma_{\min A_{z}(p)} W\right)^{k} \\
& \left.\times \Delta_{t_{j+q^{\prime}}^{l}}\left(N-\sigma_{\min A_{z^{\prime}}(p)} W\right)^{l} 1_{\left(E_{i j}^{h h^{\prime}}\right)^{c}} 1_{\left(E_{i j}^{q q^{\prime}}\right) c}\right],
\end{aligned}
$$

where $1 \leq h, h^{\prime}, q, q^{\prime} \leq k_{n}$ and either $h=q, h^{\prime}=q^{\prime}$ or

$$
\left(t_{i+h-1}^{k}, t_{i+h}^{k}\right] \cap\left(t_{j+q^{\prime}-1}^{l}, t_{j+q^{\prime}}^{l}\right] \neq \emptyset, \quad\left(t_{i+q-1}^{k}, t_{i+q}^{k}\right] \cap\left(t_{j+h^{\prime}-1}^{l}, t_{j+h^{\prime}}^{l}\right] \neq \emptyset,
$$

as otherwise the expectation vanishes. We remark that the above sum contains $O\left(k_{n}^{2}\right)$ terms. Now we follow the same strategy as in Section 6.1.3. First, we note that

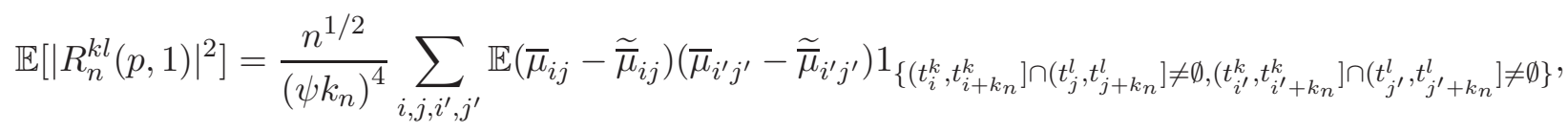

where the number of non-zero summands is $O\left(n k_{n}^{3}\right)$. Using the Cauchy-Schwarz inequality and the same approximations as at the end of Section 6.1.3, we deduce that

$$
\mathbb{E}\left[\left|R_{n}^{k l}(p, 1)\right|^{2}\right] \leq C \mathbb{E}\left[m_{p k_{n} / n, \delta}^{2}(\sigma)+\left(\frac{N_{\delta}^{\sigma}(1)}{n} \wedge 1\right)^{2}\right]
$$

for any $\delta>0$. Thus, for any fixed $p$, we have (by choosing $n$ large and then $\delta$ small) $\lim _{n \rightarrow \infty} \mathbb{E}\left[\left|R_{n}^{k l}(p, 1)\right|^{2}\right]=0$. Hence, (6.18) for any $\delta>0$, and we are done.

\subsubsection{The approximation of the mixed part}

In this section we will prove that

$$
n^{1 / 4} H Y_{k l}^{n}[X, \varepsilon]=M_{n}^{k l}(X, \varepsilon, p)+\widetilde{M}_{n}^{k l}(X, \varepsilon, p)+R_{n}^{k l}(p)
$$


where $M_{n}(X, \varepsilon, p)$ and $\widetilde{M}_{n}(X, \varepsilon, p)$ are defined by (6.6) and (6.8) respectively, $H Y_{k l}^{n}[X, \varepsilon]$ is given by (6.3) and some $R_{n}^{k l}(p)$ with (6.18) for all $\delta>0$. This proof is easier than the proofs in previous sections, because the processes $X$ and $\varepsilon$ are independent. We first show that

$$
n^{1 / 4} H Y_{k l}^{n}[D, \varepsilon]=\frac{n^{1 / 4}}{\left(\psi k_{n}\right)^{2}} \sum_{i=0}^{n_{k}-k_{n}+1} \sum_{j=0}^{n_{l}-k_{n}+1}\left(\bar{D}_{t_{i}^{k}}^{k} \bar{\varepsilon}_{t_{j}^{l}}^{l}+\bar{\varepsilon}_{t_{i}^{k}}^{k} \bar{D}_{t_{j}^{l}}^{l}\right) 1_{\left\{\left(t_{i}^{k}, t_{i+k_{n}}^{k}\right] \cap\left(t_{j}^{l}, t_{j+k_{n}}^{l}\right] \neq \emptyset\right\}}
$$

is a negligible sequence. Using Lemma 6.2 and proceeding as in the treatment of the term $\widetilde{H Y}_{k l}^{n}[D, N]$ from (6.12) we deduce that $\mathbb{E}\left[\left|H Y_{k l}^{n}[D, \varepsilon]\right|^{2}\right] \leq C n^{-1}$. Hence, $n^{1 / 4} H Y_{k l}^{n}[D, \varepsilon] \stackrel{\mathbb{P}}{\longrightarrow} 0$. Next, we obtain that

$$
\begin{aligned}
& R_{n}^{k l}(p)=n^{1 / 4} H Y_{k l}^{n}[N, \varepsilon]-M_{n}^{k l}(X, \varepsilon, p)-\widetilde{M}_{n}^{k l}(X, \varepsilon, p)+o_{\mathbb{P}}(1)
\end{aligned}
$$

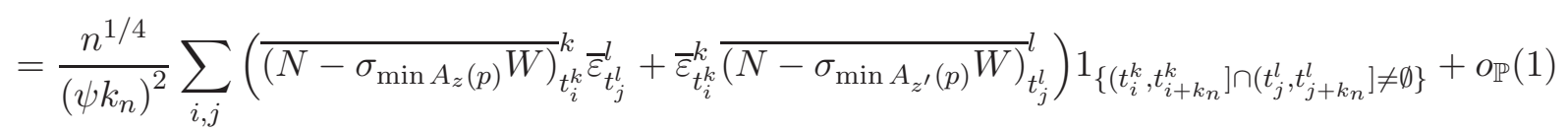

Using again Lemma 6.2, the independence between $\varepsilon$ and the components of $X$, and similar methods as for $R_{n}^{k l}(p, 1)$ in the previous section, we conclude that

$$
\mathbb{E}\left[\left|R_{n}^{k l}(p)\right|^{2}\right] \leq C \mathbb{E}\left[m_{p k_{n} / n, \delta}^{2}(\sigma)+\left(\frac{N_{\delta}^{\sigma}(1)}{n} \wedge 1\right)^{2}\right]
$$

for any $\delta>0$. Thus, for any fixed $p$, we have $\lim _{n \rightarrow \infty} \mathbb{E}\left[\left|R_{n}^{k l}(p, 1)\right|^{2}\right]=0$, and hence (6.18) for any $\delta>0$, and we are done.

\subsubsection{The noise part and the final identity}

Finally, we will show that

$$
n^{1 / 4} H Y_{k l}^{n}[\varepsilon]=M_{n}^{k l}(\varepsilon, p)+\widetilde{M}_{n}^{k l}(\varepsilon, p)+R_{n}^{k l}(p),
$$

where $M_{n}(\varepsilon, p)$ and $\widetilde{M}_{n}(\varepsilon, p)$ are defined by (6.6) and (6.8) respectively, for some $R_{n}^{k l}(p)$ with (6.18) for all $\delta>0$. This is a relatively easy exercise, because by definition we just need to prove that $n^{1 / 4} \mathbb{E}\left[H Y_{k l}^{n}[\varepsilon]\right]=o(1)$. By reordering the statistic $H Y_{k l}^{n}$ we obtain that

$$
n^{1 / 4} \mathbb{E}\left[H Y_{k l}^{n}[\varepsilon]\right]=\frac{n^{1 / 4}}{\left(\psi k_{n}\right)^{2}} \mathbb{E}\left[\sum_{i, j: t_{i}^{k}=t_{j}^{l}} a_{i j}^{k l}(n) \varepsilon_{t_{i}^{k}}^{k} \varepsilon_{t_{j}^{l}}^{l}\right]
$$

for some constants $a_{i j}^{k l}(n)$ with $\left|a_{i j}^{k l}(n)\right| \leq C$. A simple calculation shows that

$$
a_{i j}^{k l}(n)=\left(\sum_{j=0}^{k_{n}-1} g\left(\frac{j+1}{k_{n}}\right)-g\left(\frac{j}{k_{n}}\right)\right)^{2}=(g(1)-g(0))^{2}=0
$$


except for those $t_{i}^{k}$ and $t_{j}^{l}$ that are among the first and last $O\left(n^{1 / 2}\right)$ summands. Hence, $n^{1 / 4} \mathbb{E}\left[H Y_{k l}^{n}[\varepsilon]\right]=o(1)$ and we deduce that

$$
\lim _{p \rightarrow \infty} \limsup _{n \rightarrow \infty} \mathbb{P}\left(\left|n^{1 / 4} H Y_{k l}^{n}[\varepsilon]-M_{n}^{k l}(\varepsilon, p)-\widetilde{M}_{n}^{k l}(\varepsilon, p)\right|>\delta\right)=0
$$

for all $\delta>0$.

Finally, let us put things together. In Sections 6.1.3-6.1.7 we have proved the identity

$$
L^{n}=n^{1 / 4}\left(H Y^{n}-[X]\right)=M_{n}(p)+\widetilde{M}_{n}(p)+R_{n}(p)
$$

for some $R_{n}(p)$ and we have shown (see Section 6.1.2) that

$$
\lim _{p \rightarrow \infty} \limsup _{n \rightarrow \infty} \mathbb{P}\left(\left|\widetilde{M}_{n}(p)\right|+\left|R_{n}(p)\right|>\delta\right)=0
$$

for all $\delta>0$. On the other hand, we have proved in Section 6.1.1 that

$$
M_{n}(p) \stackrel{d_{s t}}{\longrightarrow} M(p)=M N\left(0, V_{p}\right) \quad \text { as } n \rightarrow \infty
$$

and, for $p \rightarrow \infty$ :

$$
V_{p} \stackrel{\mathbb{P}}{\longrightarrow} V, \quad M(p) \stackrel{\mathbb{P}}{\longrightarrow} L=M N(0, V) .
$$

This completes the proof of Theorem 3.4.

\subsection{Consistency of the variance estimators}

\subsubsection{Proof of Theorem 4.1}

It is obviously enough to prove the result for the unsymmetrized estimator

$$
\widetilde{V}_{k l, k^{\prime} l^{\prime}}^{n, 1}=\sqrt{n} \sum_{\alpha=1}^{\left[\frac{n}{\beta_{n}}\right]-1}\left(H Y_{k l}^{n}(\alpha) H Y_{k^{\prime} l^{\prime}}^{n}(\alpha)-H Y_{k l}^{n}(\alpha) H Y_{k^{\prime} l^{\prime}}^{n}(\alpha-1)\right)
$$

only, and we introduce two approximating versions of $H Y_{k l}^{n}(\alpha)$ first, namely

$$
\begin{aligned}
& \widetilde{H Y}_{k l}^{n}(\alpha)=\frac{1}{\left(\psi k_{n}\right)^{2}} \sum_{t_{i}^{k} \in B_{n}(\alpha)} \sum_{j=0}^{n_{l}-k_{n}+1} \bar{Z}(\alpha)_{t_{i}^{k}}^{k} \bar{Z}(\alpha)_{t_{j}^{l}}^{l} 1_{\left\{\left(t_{i}^{k}, t_{i+k_{n}}^{k}\right] \cap\left(t_{j}^{l}, t_{j+k_{n}}^{l}\right] \neq \emptyset\right\},} \\
& \overline{H Y}_{k l}^{n}(\alpha)=\frac{1}{\left(\psi k_{n}\right)^{2}} \sum_{t_{i}^{k} \in B_{n}(\alpha)} \sum_{j=0}^{n_{l}-k_{n}+1} \bar{Z}(\alpha-1)_{t_{i}^{k}}^{k} \bar{Z}(\alpha-1)_{t_{j}^{l}}^{l} 1_{\left\{\left(t_{i}^{k}, t_{i+k_{n}}^{k}\right] \cap\left(t_{j}^{l}, t_{j+k_{n}}^{l}\right] \neq \emptyset\right\}},
\end{aligned}
$$


where we have set

$$
\overline{Z(\alpha)}_{t_{i}^{k}}^{k}=\bar{\varepsilon}_{t_{i}^{k}}^{k}+\sum_{\nu=1}^{d} \sigma_{\frac{\alpha \beta_{n}}{n}}^{k \nu}{\overline{W^{v}}}_{t_{i}^{k}}^{k}
$$

as in (6.1), and the $W^{\nu}$ denote the independent components of the $d^{\prime}$-dimensional Brownian motion $W$. Since $\sigma$ is assumed to be an Itô semimartingale itself, the error due to replacing $\bar{Y}_{t_{i}^{k}}^{k}$ by $\overline{Z(\alpha)}_{t_{i}^{k}}^{k}$ is small: Let $t_{i}^{k} \in B_{n}(\alpha)$. Then

$$
\begin{aligned}
E\left|\bar{Y}_{t_{i}^{k}}^{k}-\overline{Z(\alpha)}_{t_{i}^{k}}^{k}\right| & =E\left|\sum_{j=1}^{k_{n}-1} g\left(j / k_{n}\right)\left(\Delta_{t_{i+j}^{k}} D^{k}+\sum_{\nu=1}^{d} \int_{\frac{i+j-1}{n}}^{\frac{i+j}{n}}\left(\sigma_{s}^{k \nu}-\sigma_{\frac{\alpha \beta_{n}}{n}}^{k \nu}\right) d W_{s}^{\nu}\right)\right| \\
& \leq C\left(\frac{k_{n}}{n}+\left(\sum_{j=1}^{k_{n}-1} g^{2}\left(j / k_{n}\right) \sum_{\nu=1}^{d} \mathbb{E}\left|\int_{\frac{i+j-1}{n}}^{\frac{i+j}{n}}\left(\sigma_{s}^{k \nu}-\sigma_{\frac{\alpha \beta_{n}}{n}}^{k \nu}\right) d W_{s}^{\nu}\right|^{2}\right)^{1 / 2}\right) \\
& \leq C\left(\frac{k_{n}}{n}+\left(k_{n} \frac{1}{n} \frac{\beta_{n}}{n}\right)^{1 / 2}\right) \leq C \frac{\sqrt{k_{n} \beta_{n}}}{n}
\end{aligned}
$$

Lemma 6.1 and Lemma 6.2 give $E\left|H Y_{k l}^{n}(\alpha)\right| \leq C \beta_{n} / n$, thus it is simple to deduce $E\left|H Y_{k l}^{n}(\alpha)-\widetilde{H Y}_{k l}^{n}(\alpha)\right| \leq$ $C\left(\beta_{n} / n\right)^{3 / 2}$, and analogously for $\overline{H Y}_{k l}^{n}(\alpha)$, so using $\eta<2 / 3$ we obtain $\widetilde{V}_{k l, k^{\prime} l^{\prime}}^{n, 1}-\bar{V}_{k l, k^{\prime} l^{\prime}}^{n, 1}=o_{\mathbb{P}}(1)$ with

$$
\bar{V}_{k l, k^{\prime} l^{\prime}}^{n, 1}=\sqrt{n} \sum_{\alpha=1}^{\left[\frac{n}{\beta_{n}}\right]}\left(\overline{H Y}_{k l}^{n}(\alpha) \overline{H Y}_{k^{\prime} l^{\prime}}^{n}(\alpha)-\overline{H Y}_{k l}^{n}(\alpha) \widetilde{H Y}_{k^{\prime} l^{\prime}}^{n}(\alpha-1)\right) .
$$

The remainder of the proof is simple now. Without loss of generality let $\beta_{n}>4 b k_{n}$ hold, so only $\overline{H Y}_{k^{\prime} l^{\prime}}^{n}(\alpha)$ and $\overline{H Y}_{k^{\prime} l^{\prime}}^{n}(\alpha+1)$ might share increments of $Y$. Then we obtain

$$
\begin{aligned}
& \sqrt{n}\left|\sum_{\alpha=1}^{\left[\frac{n}{\beta_{n}}\right]} \mathbb{E}\left(\overline{H Y}_{k l}^{n}(\alpha) \overline{H Y}_{k^{\prime} l^{\prime}}^{n}(\alpha)-\mathbb{E}\left[\overline{H Y}_{k l}^{n}(\alpha) \overline{H Y}_{k^{\prime} l^{\prime}}^{n}(\alpha) \mid \mathcal{F}_{\underline{(\alpha-1) \beta_{n}}}^{n}\right]\right)\right| \leq C \frac{\beta_{n}^{3 / 2}}{n}, \\
& \sqrt{n}\left|\sum_{\alpha=1}^{\left[\frac{n}{\beta_{n}}\right]} \mathbb{E}\left(\overline{H Y}_{k l}^{n}(\alpha) \widetilde{H Y}_{k^{\prime} l^{\prime}}^{n}(\alpha-1)-\mathbb{E}\left[\overline{H Y}_{k l}^{n}(\alpha) \widetilde{H Y}_{k^{\prime} l^{\prime}}^{n}(\alpha-1) \mid \mathcal{F}_{\frac{(\alpha-1) \beta_{n}}{n}}\right]\right)\right| \leq C \frac{\beta_{n}^{3 / 2}}{n},
\end{aligned}
$$

by conditional independence, and we are left with

$$
\bar{V}_{k l, k^{\prime} l^{\prime}}^{n, 1}=\sqrt{n} \sum_{\alpha=1}^{\left[\frac{n}{\beta_{n}}\right]} E\left[\overline{H Y}_{k l}^{n}(\alpha) \overline{H Y}_{k^{\prime} l^{\prime}}^{n}(\alpha)-\overline{H Y}_{k l}^{n}(\alpha) \widetilde{H Y}_{k^{\prime} l^{\prime}}^{n}(\alpha-1) \mid \mathcal{F}_{\left.\frac{(\alpha-1) \beta_{n}}{n}\right]+O_{\mathbb{P}}}(1) .\right.
$$

Write $V_{k l, k^{\prime} l^{\prime}}=\int_{0}^{1} r_{u} d u$, where the process $r$ is given by the right hand side of (3.4). From the same arguments as in Lemma 7.3 and Lemma 7.5 in the Appendix plus using $\eta>1 / 2$ we obtain

$$
\sqrt{n} E\left[\overline{H Y}_{k l}^{n}(\alpha) \overline{H Y}_{k^{\prime} l^{\prime}}^{n}(\alpha)-\overline{H Y}_{k l}^{n}(\alpha) \widetilde{H Y}_{k^{\prime} l^{\prime}}^{n}(\alpha-1) \mid \mathcal{F}_{\frac{(\alpha-1) \beta_{n}}{n}}\right]=\int_{\frac{\alpha \beta_{n}}{n}}^{\frac{(\alpha+1) \beta_{n}}{n}} r(u) d u+o\left(\frac{\beta_{n}}{n}\right),
$$

uniformly in $\alpha$, and the proof is complete. 


\subsubsection{Proof of Theorem 4.2}

From the proof of Theorem 3.1 we have

$$
H Y^{n}([0, s])-H Y^{n}\left(\left[0, s-l_{n}\right]\right)-\int_{s-l_{n}}^{s} \Sigma_{u} d u=o_{\mathbb{P}}\left(l_{n}\right)
$$

uniformly in $s$. Therefore the discussion on $\Psi_{n}^{k l}$ shows that we are left to prove

$$
\int_{l_{n}}^{1}\left(\frac{\int_{s-l_{n}}^{s} \Sigma_{u} d u}{l_{n}}-\Sigma_{s}\right) d s=o \mathbb{P}(1)
$$

which by left-continuity is obvious as well.

\subsubsection{Proof of Theorem 4.3}

All we need to prove is

$$
\frac{\kappa}{3 \theta \mu^{2}} \sum_{i=1}^{n-k_{n}+1}\left|\bar{Y}_{t_{i}}\right|^{4} \stackrel{\mathbb{P}}{\longrightarrow} \kappa \theta \int_{0}^{1} \frac{\sigma_{u}^{4}}{f^{\prime}(u)} d u+\frac{2 \kappa \tilde{\mu}}{\theta \mu} \Psi \int_{0}^{1} \sigma_{u}^{2} d u+\frac{\kappa \tilde{\mu}^{2}}{\theta^{3} \mu^{2}} \Psi^{2} .
$$

Since $\sigma$ is càglàd, we know from the proof of Theorem 1 in [20] that we may replace $\left|\bar{Y}_{t_{i}}\right|^{4}$ by $\left|\sigma_{t_{i}} \bar{W}_{t_{i}}+\bar{\varepsilon}_{t_{i}}\right|^{4}$ without affecting the limit. We have

$$
\frac{2 \kappa}{3 \theta \mu^{2}} \sum_{i=1}^{n-k_{n}+1} \sigma_{t_{i}}^{4} \mathbb{E}\left[\left|\bar{W}_{t_{i}}\right|^{4}\right]=\frac{2 \kappa}{\theta} \frac{k_{n}^{2}}{n^{2}} \sum_{i=1}^{n-k_{n}+1} \sigma_{t_{i}}^{4}+o_{\mathbb{P}}(1)=2 \kappa \theta \frac{1}{n} \sum_{i=1}^{n-k_{n}+1} \sigma_{t_{i}}^{4}+o_{\mathbb{P}}(1),
$$

and similar identities hold for $6\left|\bar{W}_{t_{i}}\right|^{2}\left|\bar{\varepsilon}_{t_{i}}\right|^{2}$ and $\left|\bar{\varepsilon}_{t_{i}}\right|^{4}$ as well. The result follows easily now from a Riemann approximation.

\section{Appendix}

In this final paragraph we discuss the computation of the asymptotic (conditional) variance $V_{p}$ from Theorem 6.3, which amounts to showing step (i) of its proof, and to prove convergence of $V_{p}$ to the final variance $V$ afterwards. We start with a decomposition of $\zeta_{z n}^{k l}(p)$ into a pure diffusion part, two mixed parts and a noise one, as we write

$$
\zeta_{z n}^{k l}(p)=\sum_{s=1}^{3} \zeta_{z n}^{k l}(s, p), \quad \zeta_{z n}^{k l}(s, p)=n^{1 / 4} \sum_{t_{i}^{k}, t_{j}^{l} \in B_{z}(p)}\left(\alpha_{i j}^{k l}(s, p)-\mathbb{E}\left[\alpha_{i j}^{k l}(s, p) \mid \mathcal{F}_{\min B_{z}(p)}\right]\right),
$$


with

$$
\begin{aligned}
& \alpha_{i j}^{k l}(1, p)=\frac{1}{\left(\psi k_{n}\right)^{2}}\left(\overline{\sigma_{\min B_{z}(p)} W}\right)_{t_{i}^{k}}^{k}\left(\overline{\sigma_{\min B_{z}(p)} W}\right)_{t_{j}^{l}}^{l} 1_{\left\{\left(t_{i}^{k}, t_{i+k_{n}}^{k}\right] \cap\left(t_{j}^{l}, t_{j+k_{n}}^{l}\right] \neq \emptyset\right\}}, \\
& \alpha_{i j}^{k l}(2, p)=\frac{1}{\left(\psi k_{n}\right)^{2}}\left[\left(\overline{\sigma_{\min B_{z}(p)} W}\right)_{t_{i}^{k}}^{k} \bar{\varepsilon}_{t_{j}^{l}}^{l}+\bar{\varepsilon}_{t_{i}^{k}}^{k}\left(\overline{\sigma_{\min B_{z}(p)} W}\right)_{t_{j}^{l}}^{l}\right] 1_{\left\{\left(t_{i}^{k}, t_{i+k_{n}}^{k}\right] \cap\left(t_{j}^{l}, t_{j+k_{n}}^{l}\right] \neq \emptyset\right\},}, \\
& \alpha_{i j}^{k l}(3, p)=\frac{1}{\left(\psi k_{n}\right)^{2}} \bar{\varepsilon}_{t_{i}^{k}}^{k} \bar{\varepsilon}_{t_{j}^{l}}^{l} 1_{\left\{\left(t_{i}^{k}, t_{i+k_{n}}^{k}\right] \cap\left(t_{j}^{l}, t_{j+k_{n}}^{l}\right] \neq \emptyset\right\} .} .
\end{aligned}
$$

By independence of $W$ and $\varepsilon$ it suffices to discuss

$$
V_{p}^{k l, k^{\prime} l^{\prime}}(s)=\sum_{z} \mathbb{E}\left[\zeta_{z n}^{k l}(s, p) \zeta_{z n}^{k^{\prime} l^{\prime}}(s, p) \mid \mathcal{F}_{\min B z}(p)\right]
$$

with $s=1,2,3$ only, and the final variance $V_{p}^{k l, k^{\prime} l^{\prime}}$ is the sum of the three limits in probability. Throughout each of the next subsections we also write

$$
\beta_{i j q r}^{k l k^{\prime} l^{\prime}}(s, p)=\left(\alpha_{i j}^{k l}(s, p)-\mathbb{E}\left[\alpha_{i j}^{k l}(s, p) \mid \mathcal{F}_{\min B_{z}(p)}\right]\right)\left(\alpha_{q r}^{k^{\prime} l^{\prime}}(s, p)-\mathbb{E}\left[\alpha_{q r}^{k^{\prime} l^{\prime}}(s, p) \mid \mathcal{F}_{\min B_{z}(p)}\right]\right),
$$

and we introduce the auxiliary interval

$$
\tilde{B}_{z}(p)=\left[\frac{z(p+b) k_{n}+2 b k_{n}}{n}, \frac{z(p+b) k_{n}+(p-2 b) k_{n}}{n}\right),
$$

which is slightly smaller than $B_{z}(p)$, but their sizes become close as $p$ grows eventually. Without loss of generality let $p$ be large enough for $\tilde{B}_{z}(p)$ to be non-empty.

\subsection{The contribution of the diffusion to the variance}

We begin with the pure diffusion part of the variance. By definition, we have

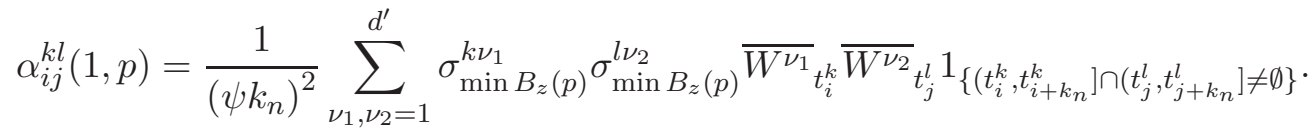

In the following we will simply write $\sigma$ instead of $\sigma_{\min B_{z}(p)}$, whenever the particular time is obvious. Recall (2.4). Setting

$$
\begin{aligned}
& F_{z, p}(k, l)=\left\{(i, j): \exists u, v \in\left\{1, \ldots, k_{n}\right\} \text { with } t_{i-u}^{k} \in B_{z}(p), t_{j-v}^{k} \in B_{z}(p)\right\}, \\
& \tilde{F}_{z, p}(k, l)=\left\{(i, j) \in F_{z, p}(k, l): t_{i}^{k} \in \tilde{B}_{z}(p)\right\}
\end{aligned}
$$

we write

$$
\sum_{t_{i}^{k}, t_{j}^{l} \in B_{z}(p)} \overline{W^{\nu_{1}}} t_{i}^{k} \overline{W^{\nu_{2}}} t_{j}^{l} 1_{\left\{\left(t_{i}^{k}, t_{i+k_{n}}^{k}\right] \cap\left(t_{j}^{l}, t_{j+k_{n}}^{l}\right] \neq \emptyset\right\}}=\sum_{(i, j) \in F_{z, p}(k, l)} c_{i j}^{n}(k, l) \Delta_{t_{i}^{k}} W^{\nu_{1}} \Delta_{t_{j}^{l}} W^{\nu_{2}}
$$


for certain numbers $c_{i j}^{n}(k, l)$ depending on the function $g$. These constants count how often and with which weight a particular product $\Delta_{t_{i}^{k}} W^{\nu_{1}} \Delta_{t_{j}^{l}} W^{\nu_{2}}$ appears in $\alpha_{i j}^{k l}(1, p)$. Let us start with a simple lemma.

Lemma 7.1 We have

$$
\begin{gathered}
\mathbb{E}\left[\zeta_{z n}^{k l}(1, p) \zeta_{z n}^{k^{\prime} l^{\prime}}(1, p) \mid \mathcal{F}_{\min } B_{z}(p)\right]=\frac{n^{1 / 2}}{\left(\psi k_{n}\right)^{4}} \sum_{(i, j) \in F_{z, p}(k, l)} \sum_{(q, r) \in F_{z, p}\left(k^{\prime}, l^{\prime}\right)} c_{i j}^{n}(k, l) c_{q r}^{n}\left(k^{\prime}, l^{\prime}\right) \\
\sum_{v_{1}, v_{2}=1}^{d}\left(\sigma^{k v_{1}} \sigma^{l v_{2}} \sigma^{k^{\prime} v_{1}} \sigma^{l^{\prime} v_{2}} \mathbb{E}\left[\Delta_{i}^{n_{k}} W^{v_{1}} \Delta_{q}^{n_{k^{\prime}}} W^{v_{1}}\right] \mathbb{E}\left[\Delta_{j}^{n_{l}} W^{v_{2}} \Delta_{r}^{n_{l^{\prime}}} W^{v_{2}}\right]\right. \\
\left.+\sigma^{k v_{1}} \sigma^{l v_{2}} \sigma^{k^{\prime} v_{2}} \sigma^{l^{\prime} v_{1}} \mathbb{E}\left[\Delta_{i}^{n_{k}} W^{v_{1}} \Delta_{r}^{n_{l^{\prime}}} W^{v_{1}}\right] \mathbb{E}\left[\Delta_{j}^{n_{l}} W^{v_{2}} \Delta_{q}^{n_{k^{\prime}}} W^{v_{2}}\right]\right) .
\end{gathered}
$$

Proof: We have to compute

$$
n^{1 / 2} \sum_{t_{i}^{k}, t_{j}^{l} \in B_{z}(p)} \sum_{t_{q}^{k^{\prime}}, t_{r}^{\prime} \in B_{z}(p)} \mathbb{E}\left[\beta_{i j q r}^{k l k^{\prime} l^{\prime}}(1, p) \mid \mathcal{F}_{\min B_{z}(p)}\right],
$$

and we begin with the conditional expectation of $\alpha_{i j}^{k l}(1, p) \alpha_{q r}^{k^{\prime} l^{\prime}}(1, p)$. Using the representations in (7.1) and (7.2) plus measurability of $\sigma$ all we have to compute is $\mathbb{E}\left[\Delta_{t_{i}^{k}} W^{\nu_{1}} \Delta_{t_{j}^{l}} W^{\nu_{2}} \Delta_{t_{q}^{k^{\prime}}} W^{\nu_{3}} \Delta_{t_{r}^{\prime}} W^{\nu_{4}}\right]$. Apply the well-known property $\mathbb{E}\left[N_{1} N_{2} N_{3} N_{4}\right]=\mathbb{E}\left[N_{1} N_{2}\right] \mathbb{E}\left[N_{3} N_{4}\right]+\mathbb{E}\left[N_{1} N_{3}\right] \mathbb{E}\left[N_{2} N_{4}\right]+\mathbb{E}\left[N_{1} N_{4}\right] \mathbb{E}\left[N_{2} N_{3}\right]$ for a (centred) normal variable $\left(N_{1}, N_{2}, N_{3}, N_{4}\right)$. As $W^{\nu_{1}}$ and $W^{\nu_{2}}$ are independent for $\nu_{1} \neq \nu_{2}$, the conditional expectation of $\alpha_{i j}^{k l}(1, p) \alpha_{q r}^{k^{\prime} l^{\prime}}(1, p)$ becomes the right hand side of (7.3) plus a third term, which is easily identified as the product of $\mathbb{E}\left[\alpha_{i j}^{k l}(1, p) \mid \mathcal{F}_{\min } B_{z}(p)\right]$ and $\mathbb{E}\left[\alpha_{q r}^{k^{\prime} l^{\prime}}(1, p) \mid \mathcal{F}_{\min B_{z}(p)}\right]$. This gives the result.

Using the previous lemma, the main part of the remainder consists in a computation of the constants $c_{i j}^{n}(k, l)$. Let us keep $i$ with $t_{i}^{k} \in B_{z}(p)$ fixed for the moment and define various auxiliary quantities, namely

$$
\tilde{j}=\left[n_{l} f_{l}\left(t_{i-k_{n}}^{k}\right)\right], \quad j^{\prime}=\left[n_{l} f_{l}\left(t_{i}^{k}\right)\right], \quad \bar{j}=\left[n_{l} f_{l}\left(t_{i+k_{n}}^{k}\right)\right] .
$$

These quantities obviously depend on $i$ and $n$, even though it does not appear in the notation, and their use is to relate observation times in the $l$ th grid to those in the $k$ th one. For example, $j^{\prime}$ is the largest index $j$ such that $t_{j}^{l}$ is left of $t_{i}^{k}$, and $\tilde{j}$ and $\bar{j}$ play similar roles. There are two observations to be made: First, in order for $c_{i j}^{n}(k, l)$ to be non-zero, the condition

$$
\tilde{j}-k_{n}+1 \leq j \leq \bar{j}+k_{n}-1
$$

has to hold. This is an easy consequence of the fact that $t_{i-k_{n}}^{k}<t_{j+k_{n}-1}^{l}$ and $t_{i+k_{n}-1}^{k}>t_{j-k_{n}}^{l}$ need to be satisfied in order for the product of the corresponding increments of $Y^{k}$ and $Y^{l}$ to appear in $H Y^{n}$. Second, it is not obvious that 
$\tilde{j}-k_{n}+1$ and $\bar{j}+k_{n}-1$ correspond to time points of $B_{z}(p)$ as well. However, by definition of $b$ we know that they do if $t_{i}^{k}$ belongs to $\tilde{B}_{z}(p)$, as for example $t_{i-k_{n}}^{k}$ lies within $\left[t_{i}^{k}-\frac{b k_{n}}{n}, t_{i}^{k}\right)$ and thus $t_{\tilde{j}-k_{n}-1}^{l} \in\left[t_{i}^{k}-\frac{2 b k_{n}}{n}, t_{i}^{k}\right)$. Let us focus on this case for a moment, as these terms are responsible for the main contribution to $V_{p}$.

Lemma 7.2 Assume that we have $t_{i}^{k} \in \tilde{B}_{z}(p)$ and recall the definition of the functions $h_{k l}$ and $\psi$ in (3.1) and (3.2). Then we have, uniformly for all $(i, j)$ that satisfy (7.4),

$$
c_{i j}^{n}(k, l)=k_{n}^{2} \psi\left(\frac{n_{l} f_{l}\left(t_{i}^{k}\right)-j}{k_{n}}, h_{k l}\left(t_{i}^{k}\right)\right)+o\left(k_{n}^{2}\right) .
$$

Proof: One singles out four cases for $j$ and computes $c_{i j}^{n}=c_{i j}^{n}(k, l)$ for each of these separately. For example,

$$
\tilde{j}-k_{n}+1 \leq j \leq \tilde{j} \quad \text { gives } \quad c_{i j}^{n}=\sum_{l_{1}=1}^{j-1-\left(\tilde{j}-k_{n}\right)} \sum_{l_{2}=\max \left(i+1-\left[n_{k} f_{k}\left(t_{j+k_{n}-l_{1}}^{l}\right)\right], 1\right)}^{k_{n}} g\left(l_{1} / k_{n}\right) g\left(l_{2} / k_{n}\right),
$$

all identities up to a possible error of (uniform) order $k_{n}$. This can be seen as follows: First, the choice of $l_{1}$ is limited, as $g\left(l_{1} / k_{n}\right)$ comes from $\bar{W}_{t_{j-l_{1}}^{l}}$ which involves $\Delta_{t_{j}^{l}}^{n_{l}} W$ as its $l_{1}$ th summand. If $l_{1}$ is small, then at least some pre-averaged statistics in the $k$ th grid starting left of $t_{\left[n_{k} f_{k}\left(t_{j+k_{n}-l_{1}}^{l}\right)\right]}^{k}$ intersect with $\bar{W}_{t_{j-l_{1}}^{l}}$ and include $\Delta_{t_{i}^{k}}^{n_{k}} W$, and those ones are responsible for $g\left(l_{2} / k_{n}\right)$. On the other hand, if $l_{1}$ is $j-\left(\tilde{j}-k_{n}\right)$ or larger, then the corresponding $\bar{W}_{t_{j-l_{1}}^{l}}$ has only empty intersections with any pre-averaged statistic in the $k$ th grid involving $\Delta_{i}^{n_{k}} W$. Similar arguments hold in the other situations, as

$$
\begin{aligned}
\tilde{j}<j \leq j^{\prime} \quad \text { gives } \quad c_{i j}^{n}=\sum_{l_{1}=1}^{k_{n}} \sum_{l_{2}=\max \left(i+1-\left[n_{k} f_{k}\left(t_{j+k_{n}-l_{1}}^{l}\right)\right], 1\right)}^{k_{n}} g\left(l_{1} / k_{n}\right) g\left(l_{2} / k_{n}\right), \\
j^{\prime}<j<\bar{j} \text { gives } c_{i j}^{n}=\sum_{l_{1}=1}^{k_{n}} \sum_{l_{2}=1}^{\min \left(k_{n}+i-1-\left[n_{k} f_{k}\left(t_{j-l_{1}}^{l}\right)\right], k_{n}\right)} g\left(l_{1} / k_{n}\right) g\left(l_{2} / k_{n}\right), \\
\bar{j} \leq j \leq \bar{j}+k_{n}-1 \text { gives } \quad c_{i j}^{n}=\sum_{l_{1}=j-\bar{j}+1}^{\min \left(k_{n}+i-1-\left[n_{k} f_{k}\left(t_{j-l_{1}}^{l}\right)\right], k_{n}\right)} g\left(l_{1} / k_{n}\right) g\left(l_{2} / k_{n}\right) .
\end{aligned}
$$

One can forget about minimum and maximum in the second sums, because $g$ vanishes outside of $[0,1]$ anyway. Have a look at the first expression now. For $l_{1} \geq j-\left(\tilde{j}-k_{n}\right)$ we obtain by monotonicity

$$
\begin{aligned}
i+1-\left[n_{k} f_{k}\left(t_{j+k_{n}-l_{1}}^{l}\right)\right] & \geq i+1-\left[n_{k} f_{k}\left(t_{j+k_{n}-\left(j-\left(\tilde{j}-k_{n}\right)\right)}^{l}\right)\right]=i+1-\left[n_{k} f_{k}\left(t_{\tilde{j}}^{l}\right)\right] \\
& \geq i+1-\left(i-k_{n}\right)=k_{n}+1 .
\end{aligned}
$$


By assumption on $g$ again we see that the sum over $l_{1}$ in the first expression for $c_{i j}^{n}$ may thus be allowed to run to $k_{n}$ as well, and a similar argument for the fourth term yields:

$$
\begin{gathered}
\tilde{j}-k_{n}+1<j \leq j^{\prime} \quad \text { gives } \quad c_{i j}^{n}=\sum_{l_{1}=1}^{k_{n}} \sum_{l_{2}=i+1-\left[n_{k} f_{k}\left(t_{j+k_{n}-l_{1}}^{l}\right)\right]}^{k_{n}} g\left(l_{1} / k_{n}\right) g\left(l_{2} / k_{n}\right), \\
j^{\prime}<j \leq \bar{j}+k_{n}-1 \quad \text { gives } \quad c_{i j}^{n}=\sum_{l_{1}=1}^{k_{n}} \sum_{l_{2}=1}^{k_{n}+i-1-\left[n_{k} f_{k}\left(t_{j-l_{1}}^{l}\right)\right]} g\left(l_{1} / k_{n}\right) g\left(l_{2} / k_{n}\right) .
\end{gathered}
$$

Also,

$$
\tilde{j}-k_{n}+1<j \leq j^{\prime} \Rightarrow k_{n}+i-1-\left[n_{k} f_{k}\left(t_{j-l_{1}}^{l}\right)\right] \geq k_{n}+i-1-\left[n_{k} f_{k}\left(t_{j^{\prime}-1}^{l}\right)\right] \geq k_{n},
$$

and with the same reasoning for the second case we obtain the global formula

$$
c_{i j}^{n}=\sum_{l_{1}=1}^{k_{n}} \sum_{l_{2}=i+1-\left[n_{k} f_{k}\left(t_{j+k_{n}-l_{1}}^{l}\right)\right]}^{k_{n}+i-1-\left[n_{k} f_{k}\left(t_{j-l_{1}}^{l}\right)\right]} g\left(l_{1} / k_{n}\right) g\left(l_{2} / k_{n}\right) .
$$

In order to simplify this expression further, we use the uniform approximation

$$
\begin{aligned}
n_{k} f_{k}\left(t_{j+k_{n}-l_{1}}^{l}\right) & =n_{k} f_{k}\left(t_{j^{\prime}}^{l}\right)+n_{k} f_{k}^{\prime}\left(t_{j^{\prime}}^{l}\right)\left(t_{j+k_{n}-l_{1}}^{l}-t_{j^{\prime}}^{l}\right)+o\left(k_{n}\right) \\
& =n_{k} f_{k}\left(t_{i}^{k}\right)+n_{k} f_{k}^{\prime}\left(t_{i}^{k}\right)\left(f_{l}^{-1}\left(\left(j+k_{n}-l_{1}\right) / n_{l}\right)-f_{l}^{-1}\left(j^{\prime} / n_{l}\right)\right)+o\left(k_{n}\right) \\
& =i+h_{k l}\left(t_{i}^{k}\right)\left(j+k_{n}-l_{1}-j^{\prime}\right)+o\left(k_{n}\right) .
\end{aligned}
$$

From Lemma 6.1, $\left|j+k_{n}-l_{1}-j^{\prime}\right| \leq C k_{n}$ holds, thus continuity of $f_{k}$ and its first derivative justifies each approximation. In the same way, $n_{k} f_{k}\left(t_{j-l_{1}}^{l}\right)=i+h_{k l}\left(t_{i}^{k}\right)\left(j-l_{1}-j^{\prime}\right)+o\left(k_{n}\right)$, and we get

$$
c_{i j}^{n}=\sum_{l_{1}=1}^{k_{n}} \sum_{l_{2}=h_{k l}\left(t_{i}^{k}\right)\left(j^{\prime}-j-k_{n}+l_{1}\right)}^{k_{n}+h_{k l}\left(t_{i}^{k}\right)\left(j^{\prime}-j+l_{1}\right)} g\left(l_{1} / k_{n}\right) g\left(l_{2} / k_{n}\right)+o\left(k_{n}^{2}\right)=k_{n}^{2} \int_{0}^{1} \int_{h_{k l}\left(t_{i}^{k}\right)\left(\frac{j^{\prime}-j}{k_{n}}-1+u\right)}^{1+h_{k l}\left(t_{k}^{k}\right)\left(\frac{j^{\prime}-j}{k_{n}}+u\right)} g(u) g(v) d v d u+o\left(k_{n}^{2}\right) .
$$

The claim can now be concluded easily.

With the aid of the preceding lemma it is easy to compute the main part of the variance due to Brownian motion. Recall (3.3) and the definition of $\tilde{F}_{z, p}(k, l)$. Set also

$$
k(z, p)=\left[n_{k} f_{k}\left(\frac{z(p+3 b) k_{n}}{n}\right)\right]+1, \quad \tilde{k}(z, p)=\left[n_{k} f_{k}\left(\frac{z(p+b) k_{n}+(p-2 b) k_{n}}{n}\right)\right]
$$

for any $k$, so $t_{k(z, p)}^{k}$ (or $t_{\tilde{k}(z, p)}^{k}$ ) is usually the smallest (or the largest) point in the $k$ th grid which lies within $\tilde{B}_{z}(p)$. Then we obtain the following result. 
Lemma 7.3 For any fixed $p$ we have

$$
\begin{aligned}
& \sum_{(i, j) \in \tilde{F}_{z, p}(k, l),(q, r) \in F_{z, p}\left(k^{\prime}, l^{\prime}\right)} c_{i j}^{n}(k, l) c_{q r}^{n}\left(k^{\prime}, l^{\prime}\right) \mathbb{E}\left[\Delta_{i}^{n_{k}} W^{v_{1}} \Delta_{q}^{n_{k^{\prime}}} W^{v_{1}}\right] \mathbb{E}\left[\Delta_{j}^{n_{l}} W^{v_{2}} \Delta_{r}^{n_{l^{\prime}}} W^{v_{2}}\right] \\
= & (p-4 b) \frac{k_{n}^{6}}{n^{2}} \gamma_{k, l, k^{\prime}, l^{\prime}}\left(t_{k(z, p)}^{k}\right)+o\left(k_{n}^{2}\right),
\end{aligned}
$$

uniformly in $z$.

Proof. The reason for restricting $(i, j)$ to the set $\tilde{F}_{z, p}(k, l)$ is that it allows us to use Lemma 7.2 to obtain approximate representations for all $c_{i j}^{n}(k, l)$ and $c_{q r}^{n}\left(k^{\prime}, l^{\prime}\right)$ that correspond to non-zero terms in the left hand side of the statement. In fact, since $t_{i}^{k}$ is within $\tilde{B}_{z}(p)$, we know from Lemma 6.1 that (essentially) any $t_{q}^{k^{\prime}}$ with a non-vanishing $\mathbb{E}\left[\Delta_{i}^{n_{k}} W^{v_{1}} \Delta_{q}^{n_{k^{\prime}}} W^{v_{1}}\right]$ lies within $\tilde{B}_{z}(p)$ as well, and thus the conditions for an application of Lemma 7.2 are satisfied. We obtain

$$
\begin{aligned}
& \sum_{(i, j) \in \tilde{F}_{z, p}(k, l),(q, r) \in F_{z, p}\left(k^{\prime}, l^{\prime}\right)} c_{i j}^{n}(k, l) c_{q r}^{n}\left(k^{\prime}, l^{\prime}\right) \mathbb{E}\left[\Delta_{i}^{n_{k}} W^{v_{1}} \Delta_{q}^{n_{k^{\prime}}} W^{v_{1}}\right] \mathbb{E}\left[\Delta_{j}^{n_{l}} W^{v_{2}} \Delta_{r}^{n_{l^{\prime}}} W^{v_{2}}\right] \\
& =\sum_{i=k(z, p)}^{\tilde{k}(z, p)} \sum_{j=\left[n_{l} f_{l}\left(t_{i-k_{n}}^{k}\right)\right]-k_{n}+1}^{\left[n_{l} f_{l}\left(t_{i+k_{n}}^{k}\right)\right]+k_{n}-1} c_{i j}^{n}(k, l) \sum_{q=\left[n_{k^{\prime}} f_{k^{\prime}}\left(t_{i-1}^{k}\right)\right]+1}^{\left[n_{k^{\prime}} f_{k^{\prime}}\left(t_{i}^{k}\right)\right]+1}\left(t_{i}^{k} \wedge t_{q}^{k^{\prime}}-t_{i-1}^{k} \vee t_{q-1}^{k^{\prime}}\right) \\
& {\left[n_{l^{\prime}} f_{l^{\prime}}\left(t_{j}^{l}\right)\right]+1} \\
& \sum_{r=\left[n_{l^{\prime}} f_{l^{\prime}}\left(t_{j-1}^{l}\right)\right]+1} c_{q r}^{n}\left(k^{\prime}, l^{\prime}\right)\left(t_{j}^{l} \wedge t_{r}^{l^{\prime}}-t_{j-1}^{l} \vee t_{r-1}^{l^{\prime}}\right)+o\left(k_{n}^{2}\right),
\end{aligned}
$$

since both expectations vanish for other choices of $q$ and $r$. Using (7.5) plus continuity of $\psi$ and $n_{l^{\prime}} f_{l^{\prime}}\left(t_{j}^{l}\right)=n_{l^{\prime}} f_{l^{\prime}}\left(t_{i}^{k}\right)+$ $h_{l^{\prime} l}\left(t_{i}^{k}\right)\left(j-n_{l} f_{l}\left(t_{i}^{k}\right)\right)+o\left(k_{n}\right)$, which can be shown in the same way as (7.6), we get

$$
c_{q r}^{n}\left(k^{\prime}, l^{\prime}\right)=c_{\left[n_{k^{\prime}} f_{k^{\prime}}\left(t_{i}^{k}\right)\right]\left[n_{l^{\prime}} f_{l^{\prime}}\left(t_{j}^{l}\right)\right]}^{n}\left(k^{\prime}, l^{\prime}\right)+o\left(k_{n}^{2}\right)=k_{n}^{2} \psi\left(h_{l^{\prime} l}\left(t_{i}^{k}\right) \frac{n_{l} f_{l}\left(t_{i}^{k}\right)-j}{k_{n}}, h_{k^{\prime} l^{\prime}}\left(t_{i}^{k}\right)\right)+o\left(k_{n}^{2}\right) .
$$

Using this approximation, we lose dependence of $c_{q r}^{n}\left(k^{\prime}, l^{\prime}\right)$ on $q$ and $r$. We conclude

$$
\begin{gathered}
\sum_{(i, j) \in \tilde{F}_{z, p}(k, l),(q, r) \in F_{z, p}\left(k^{\prime}, l^{\prime}\right)} c_{i j}^{n}(k, l) c_{r s}^{n}\left(k^{\prime}, l^{\prime}\right) \mathbb{E}\left[\Delta_{i}^{n_{k}} W^{v_{1}} \Delta_{q}^{n_{k^{\prime}}} W^{v_{1}}\right] \mathbb{E}\left[\Delta_{j}^{n_{l}} W^{v_{2}} \Delta_{r}^{n_{l^{\prime}}} W^{v_{2}}\right] \\
=k_{n}^{4} \sum_{i=k(z, p)}^{\tilde{k}_{l}(z, p)}\left(t_{i}^{k}-t_{i-1}^{k}\right) \sum_{j=\left[n_{l} f_{l}\left(t_{i-k_{n}}^{k}\right)\right]+k_{n}-1}^{k}\left(t_{j}^{l}-t_{j-1}^{l}\right) \\
\psi\left(\frac{n_{l} f_{l}\left(t_{i}^{k}\right)-j}{k_{n}}, h_{k, l}\left(t_{i}^{k}\right)\right) \psi\left(h_{l^{\prime} l}\left(t_{i}^{k}\right) \frac{n_{l} f_{l}\left(t_{i}^{k}\right)-j}{k_{n}}, h_{k^{\prime} l^{\prime}}\left(t_{i}^{k}\right)\right)+o\left(k_{n}^{2}\right) .
\end{gathered}
$$

Again a Taylor expansion gives

$$
t_{j}^{l}-t_{j-1}^{l}=\frac{1}{n_{l} f_{l}^{\prime}\left(t_{i}^{k}\right)}+o\left(n^{-1}\right)
$$


and similarly for $t_{i}^{k}-t_{i-1}^{k}$, and using (7.6) once more we obtain $n_{l} f_{l}\left(t_{i+k_{n}}^{k}\right)=n_{l} f_{l}\left(t_{i}^{k}\right)+h_{l k}\left(t_{i}^{k}\right) k_{n}+o\left(k_{n}\right)$ plus a similar result for $t_{i-k_{n}}^{k}$. Thus a Riemann approximation and continuity of all functions involved give

$$
\begin{gathered}
\sum_{(i, j) \in \tilde{F}_{z, p}(k, l),(q, r) \in F_{z, p}\left(k^{\prime}, l^{\prime}\right)} c_{i j}^{n}(k, l) c_{r s}^{n}\left(k^{\prime}, l^{\prime}\right) \mathbb{E}\left[\Delta_{i}^{n_{k}} W^{v_{1}} \Delta_{q}^{n_{k^{\prime}}} W^{v_{1}}\right] \mathbb{E}\left[\Delta_{j}^{n_{l}} W^{v_{2}} \Delta_{r}^{n_{l^{\prime}}} W^{v_{2}}\right] \\
=\frac{k_{n}^{5}}{n^{2}} \sum_{i=k(z, p)}^{\tilde{k}(z, p)} \frac{1}{m_{k} f_{k}^{\prime}\left(t_{i}^{k}\right)} \gamma_{k, l, k^{\prime}, l^{\prime}}\left(t_{i}^{k}\right)+o\left(k_{n}^{2}\right)=\frac{k_{n}^{5}}{n^{2}} \frac{\tilde{k}(z, p)-k(z, p)}{m_{k} f_{k}^{\prime}\left(t_{k(z, p)}^{k}\right)} \gamma_{k, l, k^{\prime}, l^{\prime}}\left(t_{k(z, p)}^{k}\right)+o\left(k_{n}^{2}\right) .
\end{gathered}
$$

The claim follows now from yet another Taylor expansion.

Lemma 7.3 only gives information about the variance part coming from those $t_{i}^{k}$ which belong to $\tilde{B}_{z}(p)$. For a fixed $p$ the other terms are not negligible, and in order to prove Theorem 6.3 it is necessary to show convergence of their contribution to $\mathbb{E}\left[\beta_{i j q r}^{k l k^{\prime} l^{\prime}}(1, p) \mid \mathcal{F}_{\min B_{z}(p)}\right]$ as well. This is why we need two additional results on their asymptotic behavior, which of course are similar in spirit to the preceding ones. Set

$$
\bar{k}(z, p)=\left[n_{k} f_{k}\left(\frac{z(p+b) k_{n}}{n}\right)\right]+1, \quad \hat{k}(z, p)=\left[n_{k} f_{k}\left(\frac{z(p+b) k_{n}+p k_{n}}{n}\right)\right]
$$

and let $\tilde{F}_{z, p}^{c}(k, l)$ be the complement of $\tilde{F}_{z, p}(k, l)$ in $F_{z, p}(k, l)$. As an analogue of the function $\psi$ we define

$$
\vartheta\left(s, x, y_{1}, y_{2}, y_{3}, y_{4}\right)=\int_{y_{1}}^{y_{2}} \int_{\max \left\{(u-1+s) x, y_{3}\right\}}^{\min \left\{1+x(s+u), y_{4}\right\}} g(u) g(v) d v d u
$$

also.

Lemma 7.4 Assume $(i, j) \in \tilde{F}_{z, p}^{c}(k, l)$. Then for any non-zero $c_{i j}^{n}(k, l)$ we have the uniform approximation

$$
c_{i j}^{n}(k, l)=k_{n}^{2} \vartheta\left(\frac{n_{l} f_{l}\left(t_{i}^{k}\right)-j}{k_{n}}, h_{k l}\left(t_{i}^{k}\right), \frac{j-\hat{l}(z, p)}{k_{n}}, \frac{j-\bar{l}(z, p)}{k_{n}}, \frac{i-\hat{k}(z, p)}{k_{n}}, \frac{i-\bar{k}(z, p)}{k_{n}}\right)+o\left(k_{n}^{2}\right) .
$$

Lemma 7.4 can obviously be proven in the same way as Lemma 7.2 (but with some more cases to distinguish between), and the only differences between both representations are the extra conditions on the bounds of the integrals, which arise naturally since $c_{i j}^{n}(k, l)$ is computed at the boundary of $B_{z}(p)$.

Finally, we need some additional notation. We set

$$
\begin{gathered}
\rho_{k, l, k^{\prime}, l^{\prime}}(w, x)=\frac{1}{n_{l} f_{l}^{\prime}(w)} \int_{-\left(1+h_{l k}(w)\right)}^{h_{l k}(w) x} \vartheta\left(s, h_{k l}(w), 0, h_{l k}(w) x-s, 0, x\right) \\
\vartheta\left(h_{l^{\prime} l}(w)\left(s, \frac{h_{k^{\prime} l^{\prime}}(w)}{h_{l^{\prime} l}(w)}, 0, h_{l k}(w) x-s, 0, x\right)\right) d s
\end{gathered}
$$


and

$$
\begin{aligned}
\lambda_{k, l, k^{\prime}, l^{\prime}}(w, x)= & \frac{1}{n_{l} f_{l}^{\prime}(w)} \int_{h_{l k}(w) x-1}^{\left(1+h_{l k}(w)\right)} \vartheta\left(s, h_{k l}(w), h_{l k}(w) x-s, 1, x, 1\right) \\
& \vartheta\left(h_{l^{\prime} l}(w)\left(s, \frac{h_{k^{\prime} l^{\prime}}(w)}{h_{l^{\prime} l}(w)}, h_{l k}(w) x-s, 1, x, 1\right)\right) d s .
\end{aligned}
$$

Lemma 7.5 We have

$$
\begin{gathered}
\sum_{(i, j) \in \tilde{F}_{z, p}^{c}(k, l),(q, r) \in F_{z, p}\left(k^{\prime}, l^{\prime}\right)} c_{i j}^{n}(k, l) c_{q r}^{n}\left(k^{\prime}, l^{\prime}\right) \mathbb{E}\left[\Delta_{i}^{n_{k}} W^{v_{1}} \Delta_{q}^{n_{k^{\prime}}} W^{v_{1}}\right] \mathbb{E}\left[\Delta_{j}^{n_{l}} W^{v_{2}} \Delta_{r}^{n_{l^{\prime}}} W^{v_{2}}\right] \\
=\frac{k_{n}^{6}}{n^{2}}\left(\frac{1}{m_{k} f_{k}^{\prime}\left(t_{\bar{k}(z, p)}^{k}\right)} \int_{0}^{2 b m_{k} f_{k}^{\prime}\left(t_{\bar{k}(z, p)}^{k}\right)} \rho_{k, l, k^{\prime}, l^{\prime}}\left(t_{\bar{k}(z, p)}^{k}, x\right) d x\right. \\
\left.+\frac{1}{m_{k} f_{k}^{\prime}\left(t_{\tilde{k}(z, p)}^{k}\right)} \int_{-2 b m_{k} f_{k}^{\prime}\left(t_{\tilde{k}(z, p)}^{k}\right)}^{1} \lambda_{k, l, k^{\prime}, l^{\prime}}\left(t_{\tilde{k}(z, p)}^{k}, x\right) d x\right)+o\left(k_{n}^{2}\right),
\end{gathered}
$$

uniformly in $z$.

Proof. Without loss of generality we prove the result for $\bar{k}(z, p) \leq i<k(z, p)$ only. Note by assumption on $b$ and $g$ that (7.9) reduces to

$$
c_{i j}^{n}(k, l)=k_{n}^{2} \vartheta\left(\frac{n_{l} f_{l}\left(t_{i}^{k}\right)-j}{k_{n}}, h_{k l}\left(t_{i}^{k}\right), 0, \frac{j-\bar{l}(z, p)}{k_{n}}, 0, \frac{i-\bar{k}(z, p)}{k_{n}}\right)+o\left(k_{n}^{2}\right)
$$

in this case. Mimicking the proof of Lemma 7.3 the variance part due to these terms becomes

$$
U_{z, p}^{k, l, k^{\prime}, l^{\prime}}=\sum_{i=\bar{k}(z, p)}^{k(z, p)}\left(t_{i}^{k}-t_{i-1}^{k}\right) \sum_{j=\bar{l}(z, p)}^{\left[n_{l} f_{l}\left(t_{i+k_{n}}^{k}\right)\right]+k_{n}-1}\left(t_{j}^{l}-t_{j-1}^{l}\right) c_{i j}^{n}(k, l) c_{\left[n_{k^{\prime}} f_{k^{\prime}}\left(t_{i}^{k}\right)\right]\left[n_{l^{\prime}} f_{l^{\prime}}\left(t_{j}^{l}\right)\right]}\left(k^{\prime}, l^{\prime}\right),
$$

up to an error of order $o\left(k_{n}^{2}\right)$. A similar Taylor expansion as (7.6) gives

$$
\begin{aligned}
& c_{\left[n_{k^{\prime}} f_{k^{\prime}}\left(t_{i}^{k}\right)\right]\left[n_{l^{\prime}} f_{l^{\prime}}\left(t_{j}^{l}\right)\right]}^{n}\left(k^{\prime}, l^{\prime}\right) \\
= & k_{n}^{2} \vartheta\left(h_{l^{\prime} l}\left(t_{i}^{k}\right)\left(\frac{n_{l} f_{l}\left(t_{i}^{k}\right)-j}{k_{n}}, \frac{h_{k^{\prime} l^{\prime}}\left(t_{i}^{k}\right)}{h_{l^{\prime} l}\left(t_{i}^{k}\right)}, 0, \frac{j-\bar{l}(z, p)}{k_{n}}, 0, \frac{i-\bar{k}(z, p)}{k_{n}}\right)\right)+o\left(k_{n}^{2}\right) .
\end{aligned}
$$

Using (7.8) and a Riemann sum argument we obtain

$$
\begin{aligned}
U_{z, p}^{k, l, k^{\prime}, l^{\prime}}= & k_{n}^{5} \sum_{i=\bar{k}(z, p)}^{k(z, p)}\left(t_{i}^{k}-t_{i-1}^{k}\right) \frac{1}{n_{l} f_{l}^{\prime}\left(t_{i}^{k}\right)} \int_{-\left(1+h_{l k}\left(t_{i}^{k}\right)\right)}^{\frac{n_{l} f_{l}\left(t_{i}^{k}\right)-\bar{l}(z, p)}{k_{n}}} \\
& \vartheta\left(s, h_{k l}\left(t_{i}^{k}\right), 0, \frac{n_{l} f_{l}\left(t_{i}^{k}\right)-\bar{l}(z, p)}{k_{n}}-s, 0, \frac{i-\bar{k}(z, p)}{k_{n}}\right) \\
& \vartheta\left(h_{l^{\prime} l}\left(t_{i}^{k}\right)\left(s, \frac{h_{k^{\prime} l^{\prime}}\left(t_{i}^{k}\right)}{h_{l^{\prime} l}\left(t_{i}^{k}\right)}, 0, \frac{n_{l} f_{l}\left(t_{i}^{k}\right)-\bar{l}(z, p)}{k_{n}}-s, 0, \frac{i-\bar{k}(z, p)}{k_{n}}\right)\right) d s+o\left(k_{n}^{2}\right) .
\end{aligned}
$$


The final step differs from the previous proof, as the dependence on $i$ is more involved now. We use continuity to obtain

$$
\frac{n_{l} f_{l}\left(t_{i}^{k}\right)-\bar{l}(z, p)}{k_{n}}=\frac{n_{l} f_{l}\left(t_{i}^{k}\right)-n_{l} f_{l}\left(t_{\bar{k}(z, p)}^{k}\right)}{k_{n}}+o(1)=h_{l k}\left(t_{\bar{k}(z, p)}^{k} \frac{i-\bar{k}(z, p)}{k_{n}}+o(1),\right.
$$

and applying (7.8) on $\left(t_{i}^{k}-t_{i-1}^{k}\right)$ plus replacing each $t_{i}^{k}$ by $t_{\bar{k}(z, p)}^{k}$ due to continuity again, we derive

$$
U_{z, p}^{k, l, k^{\prime}, l^{\prime}}=\frac{k_{n}^{6}}{n^{2}} \frac{1}{m_{k} f_{k}^{\prime}\left(t_{\bar{k}(z, p)}^{k}\right)} \int_{0}^{\frac{k(z, p)-\bar{k}(z, p)}{k_{n}}} \rho_{k, l, k^{\prime}, l^{\prime}}\left(t_{\bar{k}(z, p)}^{k}, x\right) d x+o\left(k_{n}^{2}\right) .
$$

The claim can now be obtained easily.

It is obviously possible to replace $\tilde{k}(z, p)$ and $\bar{k}(z, p)$ in (7.10) and (7.11) by $k(z, p)$ without affecting the approximation error. We set

$$
\begin{aligned}
\varphi_{k, l, k^{\prime}, l^{\prime}}(p, w)=(p-4 b) \gamma_{k, l, k^{\prime}, l^{\prime}}(w) & +\frac{1}{m_{k} f_{k}^{\prime}(w)} \int_{0}^{2 b m_{k} f_{k}^{\prime}(w)} \rho_{k, l, k^{\prime}, l^{\prime}}(w, x) d x \\
& +\frac{1}{m_{k} f_{k}^{\prime}(w)} \int_{-2 b m_{k} f_{k}^{\prime}(w)}^{1} \lambda_{k, l, k^{\prime}, l^{\prime}}(w, x) d x,
\end{aligned}
$$

and it is simple now to derive the following theorem which concludes this section.

Theorem 7.6 We have

$$
\begin{aligned}
V_{p}^{k l, k^{\prime} l^{\prime}}(1) & =\sum_{z} \mathbb{E}\left[\zeta_{z n}^{k l}(1, p) \zeta_{z n}^{k^{\prime} l^{\prime}}(1, p) \mid \mathcal{F}_{\min B_{z}(p)}\right] \\
& =\frac{\theta}{p \psi^{4}} \int_{0}^{1}\left(\varphi_{k, l, k^{\prime}, l^{\prime}}(p, w) \Sigma_{w}^{k k^{\prime}} \Sigma_{w}^{l l^{\prime}}+\varphi_{k, l, l^{\prime}, k^{\prime}}(p, w) \Sigma_{w}^{k l^{\prime}} \Sigma_{w}^{l k^{\prime}}\right) d w+o_{\mathbb{P}}(1) .
\end{aligned}
$$

For $p \rightarrow \infty$, we conclude

$$
V_{p}^{k l, k^{\prime} l^{\prime}}(1) \stackrel{\mathbb{P}}{\longrightarrow} \frac{\theta}{\psi^{4}} \int_{0}^{1}\left(\gamma_{k, l, k^{\prime}, l^{\prime}}(w) \Sigma_{w}^{k k^{\prime}} \Sigma_{w}^{l l^{\prime}}+\gamma_{k, l, l^{\prime}, k^{\prime}}(w) \Sigma_{w}^{k l^{\prime}} \Sigma_{w}^{l k^{\prime}}\right) d w
$$

which equals the pure diffusion part of (3.4).

\subsection{The contribution of the remaining parts to the variance}

In this final subsection we give some ideas on how to obtain formulas for $V_{p}^{k l, k^{\prime} l^{\prime}}(2)$ and $V_{p}^{k l, k^{\prime} l^{\prime}}(3)$, from which Theorem 6.3 (and thus in turn Theorem 3.1) can be concluded.

The main intuition in both cases it that one obtains representations for $\alpha_{i j}^{k l}(2, p)$ and $\alpha_{i j}^{k l}(3, p)$ which are closely related to (7.2) in the sense that those constants $\bar{c}_{i j}^{n}(k, l)$ and $\widetilde{c}_{i j}^{n}(k, l)$, say, can be treated in the same way as in Lemma 
7.2 and Lemma 7.4. In fact, the only difference is that $g\left(l_{1} / k_{n}\right)$ sometimes has to be replaced by $\left(-1 / k_{n}\right) g^{\prime}\left(l_{1} / k_{n}\right)$, since $\left(g\left(l_{1} / k_{n}\right)-g\left(\left(l_{1}+1\right) / k_{n}\right)\right) \varepsilon_{t_{j}^{l}}^{l}$ plays the role of $g\left(l_{1} / k_{n}\right) \Delta_{j}^{n_{l}} W$ now, and so the approximating functions in a version of Lemma 7.2 naturally become $\bar{\psi}$ and $\widetilde{\psi}$ from (3.2).

Also, Lemma 7.3 and Lemma 7.5 have expressions in this context, but the first difference is that one does not sum

over all $(i, j)$ and $(p, q)$ now, but only over those for which $t_{j}^{l}$ and $t_{r}^{l^{\prime}}$, say, coincide, as otherwise $\mathbb{E}\left[\varepsilon_{t_{j}^{l}}^{l} \varepsilon_{t_{r}^{l^{\prime}}}^{l^{\prime}}\right] \neq 0$ is not satisfied. Second,

$$
t_{j}^{l}-t_{j-1}^{l}=\frac{1}{n_{l} f_{l}^{\prime}\left(t_{i}^{k}\right)}+o\left(n^{-1}\right)
$$

is not included in the sum anymore, as this term came from an increment of Brownian motion. This explains the need for the additional terms $m_{l l^{\prime}} f_{l l^{\prime}}^{\prime}$ in $\bar{\gamma}$ and $\widetilde{\gamma}$, as the Riemann approximation otherwise does not hold.

\section{References}

[1] Aldous, D.J., and Eagleson, G.K. (1978): On mixing and stability of limit theorems. Annals of Probability 6(2), $325-331$.

[2] Barndorff-Nielsen, O.E., S.E. Graversen, J. Jacod, M. Podolskij, N. Shephard (2006): A central limit theorem for realised power and bipower variations of continuous semimartingales. In: Yu. Kabanov, R. Liptser and J. Stoyanov (Eds.), From Stochastic Calculus to Mathematical Finance. Festschrift in Honour of A.N. Shiryaev, Heidelberg: Springer, 2006, 33-68.

[3] Barndorff-Nielsen, O. E., P. R. Hansen, A. Lunde, and N. Shephard (2008): Designing realised kernels to measure the ex-post variation of equity prices in the presence of noise. Econometrica 76(6), 1481-1536.

[4] Barndorff-Nielsen, O. E., P. R. Hansen, A. Lunde, and N. Shephard (2010): Multivariate realised kernels: Consistent positive semi-definite estimators of the covariation of equity prices with noise and non-synchronous trading. To appear in Journal of Econometrics.

[5] Bandi, F. M., and J. R. Russell (2006): Separating microstructure noise from volatility. Journal of Financial Economics 79(3), 655-692.

[6] Bibinger, M. (2011): Efficient covariance estimation for asynchronous noisy high-frequency data. Scandinavian Journal of Statistics 38, 23-45. 
[7] Christensen, K., S. Kinnebrock and M. Podolskij (2010): Pre-averaging estimators of the ex-post covariance matrix in noisy diffusion models with non-synchronous data. Journal of Econometrics 159, 116-133.

[8] Christensen, K., R. Oomen and M. Podolskij (2010): Realised quantile-based estimation of the integrated variance. Journal of Econometrics 159(1), 74-98.

[9] Delbaen, F., and W. Schachermayer (1994): A general version of the fundamental theorem of asset pricing. Mathematische Annalen 300, 463-520.

[10] Glassermann, P. (2004): Monte Carlo Methods in Financial Engineering, Springer-Verlag: Berlin.

[11] Gloter, A. and J. Jacod (2001): Diffusions with measurement errors. II-Optimal estimators. ESAIM 5, 243-260.

[12] Hayashi, T., and N. Yoshida (2005): On covariance estimation of non-synchronously observed diffusion processes. Bernoulli 11(2), 359-379.

[13] Jacod, J. (1997): On continuous conditional Gaussian martingales and stable convergence in law. Seminaire de Probabilites XXXI, 232-246.

[14] Jacod, J. (2008): Asymptotic properties of realized power variations and related functionals of semimartingales. Stochastic Processes and Their Applications, 118, 517-559.

[15] Jacod, J., Y. Li, P. Mykland, M. Podolskij and M. Vetter (2009): Microstructure noise in the continuous case: the pre-averaging approach. Stochastic Processes and Their Applications 119, 2249-2276.

[16] Jacod, J., M. Podolskij and M. Vetter (2010): Limit theorems for moving averages of discretized processes plus noise. Annals of Statistics 38(3), 1478-1545.

[17] Jacod, J. and A.N. Shiryaev (2003): Limit Theorems for Stochastic Processes, 2d ed., Springer-Verlag: Berlin.

[18] Mykland, P. (2010): A Gaussian Calculus for Inference from High Frequency Data. To appear in Annals of Finance.

[19] Palandri, A. (2006): Consistent realized covariance for asynchronous observations contaminated by market microstructure noise. Working paper.

[20] Podolskij, M. and M. Vetter (2009): Bipower-type estimation in a noisy diffusion setting. Stochastic Processes and Their Applications 119, 2803-2831. 
[21] Podolskij, M. and M. Vetter (2009): Estimation of volatility functionals in the simultaneous presence of microstructure noise and jumps. Bernoulli 15(3), 634-658.

[22] Renyi, A. (1963): On stable sequences of events. Sankhya A 25, 293-302.

[23] Zhang, L. (2006): Efficient estimation of stochastic volatility using noisy observations: A multi-scale approach. Bernoulli 12(6), 1019-1043.

[24] Zhang, L., P. A. Mykland, and Y. Aït-Sahalia (2005): A tale of two time scales: determining integrated volatility with noisy high-frequency data. Journal of the American Statistical Association 100(472), 1394-1411. 

